\title{
The Sierra Norte-Ambargasta batholith: Late EdiacaraneEarly Cambrian magmatism associated with Pampean transpressional tectonics
}

\author{
Noelia F. lannizzotto ${ }^{a, \star}$, Carlos W. Rapela ${ }^{a}$, Edgardo G.A. Baldo ${ }^{b}$, Carmen Galindo ${ }^{c}$, C.M. Fanning ${ }^{d}$, \\ Robert J. Pankhurst ${ }^{\mathrm{e}}$

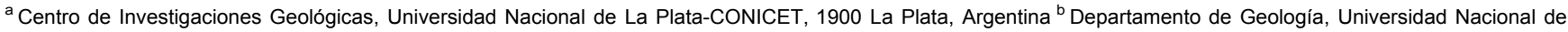 \\ Córdoba, 5000 Córdoba, Argentina \\ ${ }^{\mathrm{c}}$ Departamento de Petrología y Geoquímica, Universidad Complutense-IGEO (CSIC), 28040 Madrid, Spain ${ }^{\mathrm{d}}$ Research School of Earth Sciences, The Australian National \\ University, Canberra, Australia

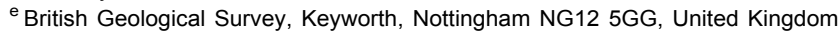

\begin{abstract}
A b s tract
The Sierra Norte-Ambargasta batholith is one of the largest plutonic expressions of the Pampean orogeny in western Argentina. A thorough petrographic, geochemical, isotopic ( $\mathrm{Sr}$ and $\mathrm{Nd}$ ) and geochronological (UePb SHRIMP) study is reported. The batholith comprises granitoid rocks that may be subdivided into those affected by Pampean D2 dextral shearing and mylonization and those emplaced after deformation had ceased; representative samples gave UePb zircon ages of $537 \pm 4 \mathrm{Ma}$ and $530 \pm 4 \mathrm{Ma}$ respectively. The earlier, dominant, group were derived largely from metaluminous calc-alkaline subduction-related magmas, whereas the late granites are peraluminous. However, all have relatively high initial ${ }^{87} \mathrm{Sr} /{ }^{86} \mathrm{Sr}$ ratios $(0.706$ to at least 0.710 , strongly negative $\varepsilon N d t$ values (-1.7 to -5.9) and, in some cases inherited $600 \mathrm{Ma}$ and $970 \mathrm{Ma}$ zircon, similar to the isotopic and zircon provenance seen in the metamorphic host rocks. A high degree of contamination of the magmas, possibly anatexis in the case of the post-mylonite granite, is related to emplacement during the latestage transpressional docking of the Pampean terrane against the Rio de la Plata craton. The absence of detrital zircon derived from the craton in either the Pampean metasedimentary host rocks or the batholith supports this collisional model for the Pampean orogen.
\end{abstract}

Keywords: Sierra Norte, Sauce Puncoelschilín mylonitic belt U/Pb SHRIMP, zircon dating, Cambrian

1.

\section{Introduction}

The pre-Late Palaeozoic Sierra Norte-Ambargasta batholith in

the Eastern Sierras Pampeanas of Argentina (Fig. 1) is located in the easternmost uplifted block above the Pacific flat-slab subduction segment, at $27^{\circ} \mathrm{S}, 33^{\circ} 30^{\prime} \mathrm{W}$. Notable previous studies are those of Methol (1958), Lucero Michaut (1969, 1979), Gordillo and Lencinas (1979), Bonalumi (1988), Rapela et al. (1991), Gordillo (1996), Kirschbaum et al. (1997), Lira et al. (1997), Baldo et al. (1998), Candiani et al. (2001), Miró and Sapp (2001), Koukharsky et al. (2003), Leal et al. (2003), Martino (2003) and Llambías et al.(2003). Other recent geochronological and structural studies have been reported by Schwartz et al. (2008), Von Gosen and Prozzi (2010) and Siegesmund et al. (2010).

\footnotetext{
* Corresponding author. Centro de Investigaciones Geológicas, Universidad Nacional de La Plata-CONICET, Calle 1 Nro 644, La Plata, B1900TAC Buenos

Aires, Argentina. Tel.: p54 0021421 5677; fax: p54 02214827560.

E-mail addresses: noelia@cig.museo.unlp.edu.ar,

Noelia.Iannizzotto@gmail.com (N.F. lannizzotto).
}

The aim of this paper is to present a thorough field and petrological description of the rocks and their deformational setting, and to combine these observations with new UePb SHRIMP zircon crystallization and provenance ages, isotopic and whole-rock geochemical data. The study is focused on the granitic rocks and their relation to large dextral mylonitic zones through a detailed study of the southern sector of the batholith.

The importance of this work is in constraining the timing of Pampean orogenic magmatism and deformation in the Eastern Sierras Pampeanas, and in assessing the role of transpressional belts during the amalgamation of southwest 2. Gondwana.

Geological setting

The Sierras Pampeanas were originally sub-divided into

Western and Eastern sectors according to their dominant lithology (Caminos, 1972) ( Fig. 1), whereas modern geochronological studies demonstrate that they correspond to different geological provinces. The Western Sierras Pampeanas are dominated by $1030 \mathrm{e} 1330 \mathrm{Ma}$ ("Grenville-age") igneous and metamorphic rocks, intruded by 


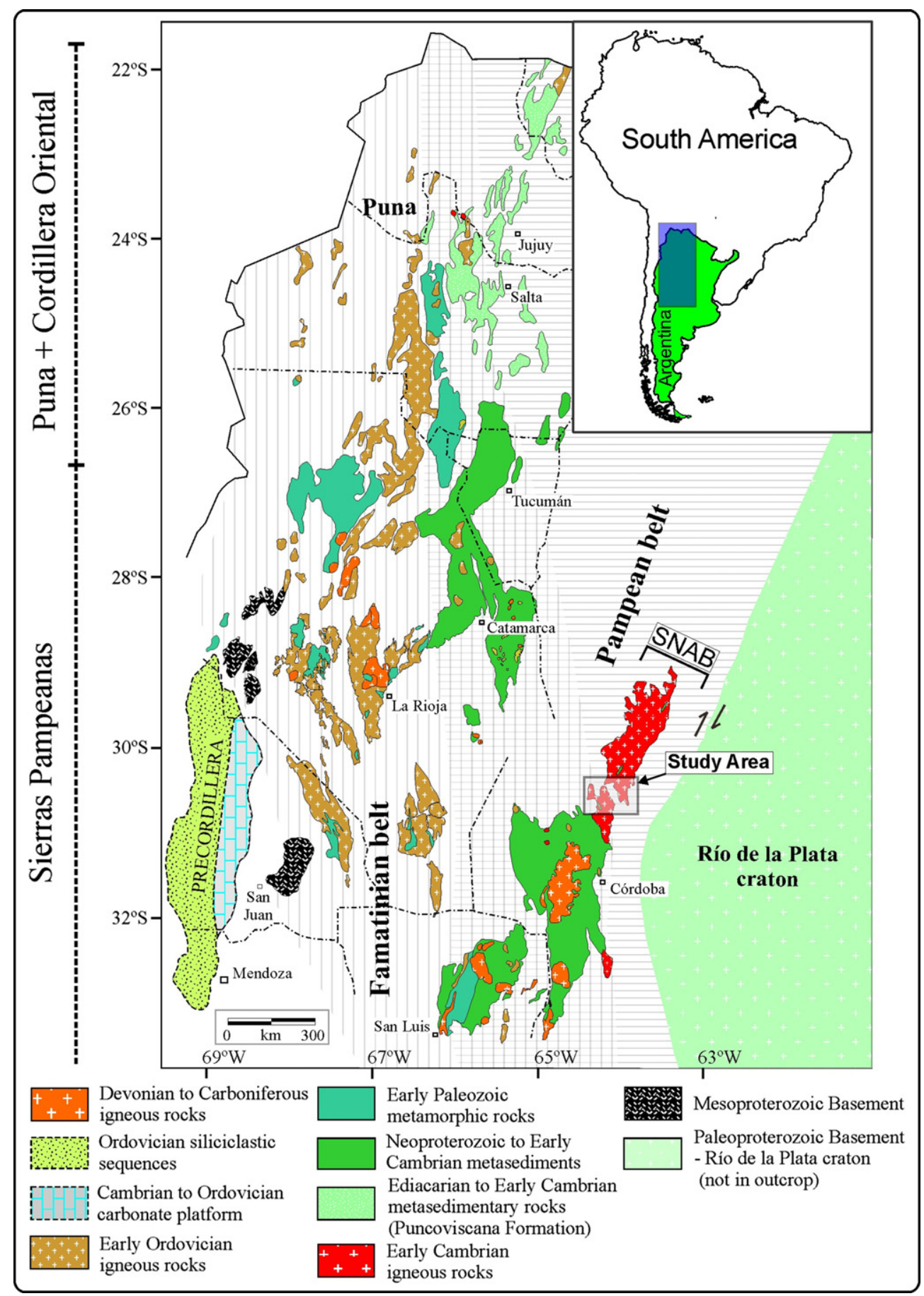

Fig. 1. Geological sketch map showing the Sierra Norte-Ambargasta batholith (SNAB) in a regional context; study area indicated.

relatively scarce Ordovician granites of the Famatinian cycle (e.g., Sato et al., 2003; Rapela et al., 2010 and references therein). The Eastern Sierras Pampeanas are characterized instead by a low- to high-grade Late Ediacaran to Early Palaeozoic basement, intruded by voluminous granitic batholiths and plutons of the Late NeoproterozoiceCambrian (Pampean), Ordovician (Famatinian) and DevonianeCarboniferous (Achalian) cycles (Pankhurst and Rapela, 1998; Dahlquist et al., 2006).

The earliest tectono-metamorphic event recognized in the Eastern Cordillera, Puna and northwestern Sierras Pampeanas is known as the Pampean Cycle or Pampean orogeny (Aceñolaza et al.,
1990 and references therein). The oldest and best-studied unit of this basement is the Puncoviscana Formation (Turner, 1960), a thick sequence $(>2000 \mathrm{~m})$ of low-grade metapelites and turbidites interbedded with minor felsic tuffs, first deposited in the Late Ediacaran (Omarini et al., 1999), but mainly in the Early Cambrian, and folded still within the Early Cambrian (537e523 Ma, Escayola et al., 2011 and references therein). The metasedimentary rocks of the northwestern Sierras Pampeanas are considered to be higher-grade equivalents of this formation (Toselli, 1990; Willner, 1990).

In the southern sector of the Eastern Sierras Pampeanas (Sierras de Córdoba, Fig. 1), Rapela et al. (1998a) demonstrated that Early 
Cambrian deformation, coeval granulite facies metamorphism and S2 deformation correspond to low structural levels of the Pampean orogeny. Isotopic and structural studies have confirmed correlation of metasedimentary sequences in the Sierra de San Luis, Sierras de Córdoba and Sierra Norte (Fig. 1) with the Puncoviscana Formation (Schwartz and Gromet, 2004; Steenken et al., 2004; Von Gosen and Prozzi, 2010).

The study area is in the southern sector of Sierra Norte de Córdoba at $30^{\circ} 24^{\circ} \mathrm{e} 30^{\circ} 46^{\circ} \mathrm{S}$ and $64^{\circ} \mathrm{We} 64^{\circ} 35^{\circ} \mathrm{W}$, near the eastern subsurface limit of the Eastern Sierras Pampeanas (Fig. 1). Its largest geomorphological features are the Sierra de La Higuerita, Sierra de Ischilín and Sierra de Macha (Fig. 2).

The igneous rocks of the Sierra Norte-Ambargasta batholith can be subdivided according to their relationship to the mylonitic deformation. The study area is dominated by pre-/syn-mylonitic plutons, consisting of biotite \pm hornblende metaluminous to slightly peraluminous rocks that were emplaced prior to and during the initial stages of compressive deformation with a dextral component (D1). Subsequently, these rocks were affected by the penetrative dextral deformation (D2) that formed major mylonitic belts near Ischilín and Sauce Punco. Subordinate, post-mylonitic, muscoviteebiotite leucogranites that intruded the mylonitic belt and previous units include the Villa Albertina granite.

\section{The host rocks: La Falda metamorphic complex}

The sequence of clastic metamorphic rocks that host the granites of Sierra Norte was considered a time equivalent of the Puncoviscana Formation by Von Gosen and Prozzi (2010). In the southern Sierra Norte-Ambargasta batholith, the metamorphic country rock occurs as kilometre-sized septa or xenoliths with sharp contacts against the pre-/syn-mylonitic granites and sharp to transitional contacts with the post-mylonitic granites (Gordillo et al., 1997; lannizzotto, 2010). The enclaves vary in size from centimetres to tens of metres. The foliation strike and dip of the xenoliths in the pre-/syn-mylonitic granites are very variable. In the study area, the metamorphic rocks are composed of paragneiss, schist and subordinate marble and amphibolite, which were all assigned to the La Falda metamorphic complex by Lyons et al. (1997).

The paragneisses are grey banded gneisses or migmatites with quartzo-feldspathic leucosomes and grano-lepidoblastic textures. They are composed of quartz, plagioclase, biotite, garnet \pm sillimanite and secondary muscovite, with accessory opaque minerals, apatite and zircon. The mineralogy and texture suggest a pelitic sedimentary protolith.

The green to dark brown garnet-mica schist consists of biotite ( $\mathrm{XMg} \mathrm{0.44,} \mathrm{TiO}_{2} 1.2 \mathrm{e} 1.6 \%$ ), quartz and less abundant plagioclase, $\mathrm{K}$ feldspar and muscovite; garnet (Alm (m4e66, $_{\text {S }}$ P 20e22, P y 10e12, $\mathrm{Grs}_{0.8 \mathrm{e}}$.0) is the principal accessory mineral and tourmaline is a very minor accessory. Some samples exhibit non-coaxial defor-mation, evidenced by mica fish and ribbons of quartz.

The marble is white to pale blue and medium- to coarse-grained granoblastic, consisting of calcite, accessory epidote, garnet (grossular), biotite, diopside and opaque minerals. In contact with acidic intrusive rocks, the marbles were metamorphosed to skarns with pink zoisite, epidote and grossular.

The dark green amphibolite is granular in texture and medium-to coarse-grained. Microscope observations indicate abundant hornblende and plagioclase, biotite, quartz, epidote, apatite and

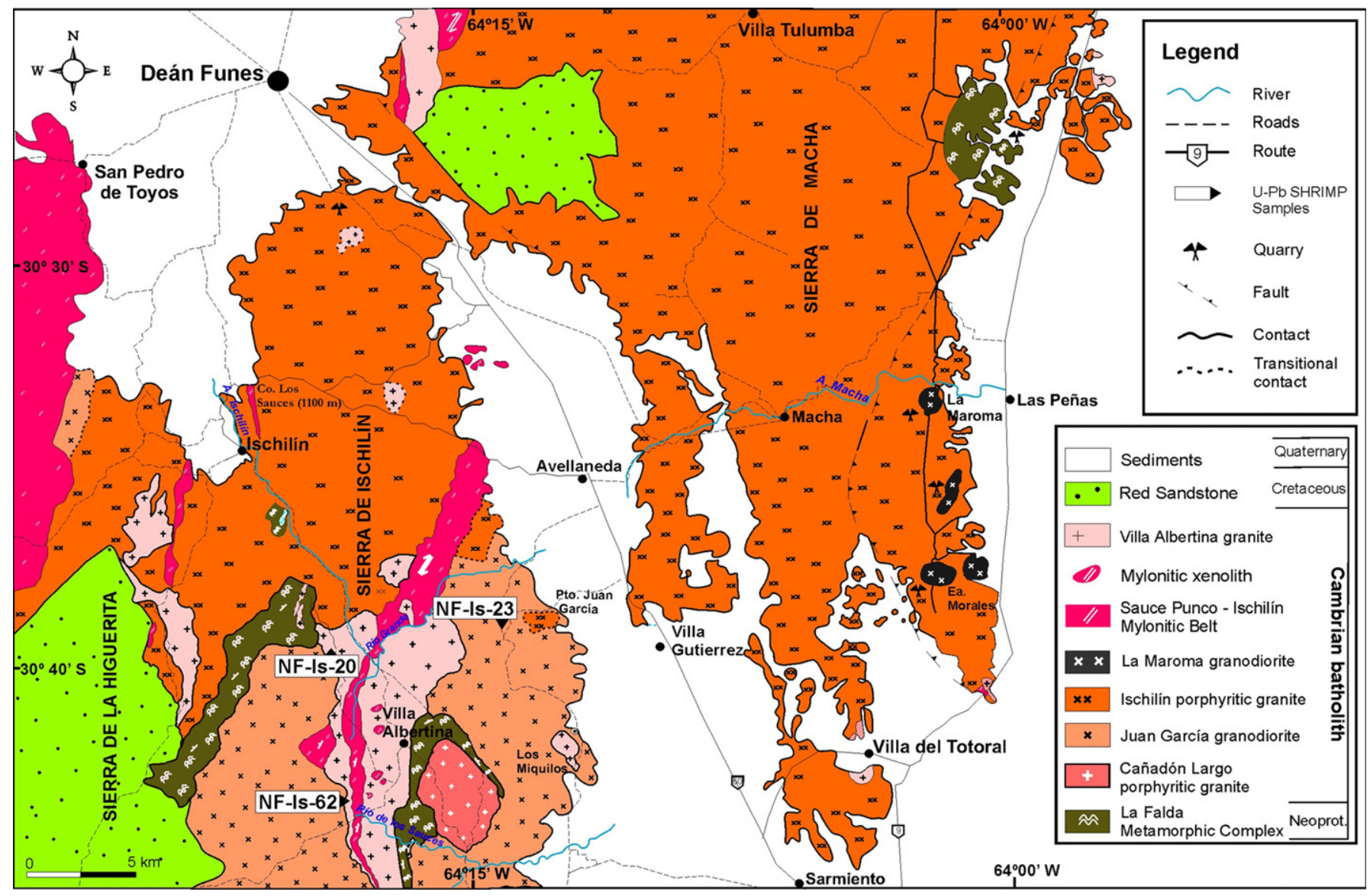

Fig. 2. Geological map of the southern Sierra Norte-Ambargasta batholith, based on Gordillo (1996), Candiani et al. (2001) and recent mapping of lannizzotto (2010). 
opaque minerals. The amphibole exhibits a tendency to develop large poikilitic crystals associated with contact metamorphism; the poikiloblasts include anhedral to subhedral microlites of plagioclase and anhedral amphibole.

\section{Sierra Norte-Ambargasta batholith}

The southern sector of the batholith (Fig. 2) consists of five plutonic units, which range in composition from tonalite to syenogranite, although the lithology is dominated by monzogranites and granodiorites; principal compositional features are summar-ised in Table 1. There are also three types of subordinate dyke. The plutons have been subdivided according to their temporal and spatial relationship to the Sauce Puncoelschilín mylonitic belt (Fig. 2). Most were affected by dextral deformation, or were emplaced contemporaneously with it, and are accordingly classi-fied as pre-/ syn-mylonitic units, whereas one plutonic unit is recognized as postmylonitic.

\subsection{Pre-/syn-mylonitic units}

The Ischilín porphyritic granite comprises the largest pluton in the southern Sierra Norte-Ambargasta batholith, extending from the Sierra de Macha to the sierras of Ischilín and La Higuerita (Fig. 2). The granite was defined by lannizzotto (2010) and includes the porphyritic biotitic monzogranite of Candiani et al. (2001) and the Macha granite of Kirschbaum et al. (1997). Locally it exhibits a NNEtrending magmatic foliation, dipping to the east and defined by the alignment of biotite, feldspar phenocrysts and mafic enclaves. The $1200 \mathrm{~km}^{2}$ pluton exhibits both sharp and transitional contacts with the other igneous units: contact with the Juan García granodiorite is transitional and occurs over a few metres, while that with the La Maroma granodiorite and the leucocratic bodies of the Villa Albertina granite is sharp. There is no exposed contact with the Cañadón Largo porphyritic granite, from which it is distin-guished by its mineralogy (Table 1). In the southern sector of Sierra de Ischilín, the pluton is truncated by the Sauce Puncoelschilín mylonitic belt and is intruded by the Villa Albertina granite (Gordillo, 1996; lannizzotto, 2010). Microgranular mafic enclaves usually exhibit either a sharp contact or have a biotite reaction rim, whereas metamorphic basement enclaves exhibit sharp contacts. The Ischilín porphyritic granite is pink and varies in composition from granodiorite to monzogranite (Fig. 3; Table 1). The texture can be microporphyritic, almost seriate, as in the sierras of Ischilín and La Higuerita (Fig. 2), with K-feldspar phenocrysts up to $1 \mathrm{~cm}$ in length, or mega-porphyritic, as in the Sierra de Macha, where the phenocrysts can reach $10 \mathrm{~cm}$. A mylonitic foliation is observed, especially along the contact area with the mylonitic belt.

The Cañadón Largo porphyritic granite was mapped as an independent pluton by Gordillo (1996). It is a $25 \mathrm{~km}^{2}$ sub-circular body, partly mylonitized and associated with one of the largest blocks of metamorphic basement in the study area (Fig. 2). The outcrops comprise altered pink porphyritic rocks, with microcline pheno-crysts up to $2 \mathrm{~cm}$ in a coarse-grained matrix of quartz, plagioclase, microcline and biotite, and with elongated mafic enclaves. Accessory garnet distinguishes this facies from the rest of the pre-/synmylonitic units (Table 1). A mylonitic foliation is defined by K-feldspar porphyroclasts, incipient quartz ribbons and alignment of biotite. The associated aplites are also commonly affected by ductile deformation.

The Juan García granodiorite (Gordillo, 1996) crops out in the Sierra de Ischilín as two large bodies of $10 \mathrm{~km}$ in length and up to 7 $\mathrm{km}$ in width, separated by the Sauce Puncoelschilín mylonitic belt and the Villa Albertina granite (Fig. 2). Outcrops appear as isolated rounded blocks. Within the Ischilín porphyritic granite, there are $50 \mathrm{e} 200 \mathrm{~m}$ sectors free of K-feldspar phenocrysts that closely resemble the Juan García granodiorite, suggesting that the two units are coeval and closely related. In terms of modal classi-fication, the Juan García granodiorite is dominated by granodiorites and monzogranites, with scarce tonalites (Fig. 3) intruded by pink aplites. It is grey to greenish grey, and has a medium grain size. There are abundant mafic microgranular enclaves and scarce metamorphic basement xenoliths with sharp contacts. A sharp contact with the Villa Albertina granite can be seen along the Río de Los Sauces (Fig. 2), where thin leucocratic dykes cutting the Juan García granodiorite could be related to the main body of the Villa Albertina granite. Gordillo (1996) recognised a biotiteehornblende Río de Los Sauces granodiorite, which is now considered as a facies variation. The magmatic foliation strikes $355^{\circ} / 44^{\circ} \mathrm{E}$ t o $304^{\circ} / 40^{\circ} \mathrm{E}$, with mafic enclaves orientated parallel to it.

The La Maroma granodiorite crops out discontinuously over 25 $\mathrm{km}(\mathrm{NeS})$ in the southeast sector of Sierra de Macha (Fig. 2). It was mentioned first by Pastore and Methol (1953) who identified small intrusive bodies of quartz diorite. González et al. (1985) described these bodies as dykes or aligned stocks along the eastern slope of the range. Kirschbaum et al. (1997) gave this unit its name and observed a sharp contact with the Macha granite (the Ischilín porphyritic granite of this work). The rocks are porphyritic biotiteehornblende granodiorites and tonalites with white plagioclase phenocrysts (up to $5 \mathrm{~cm}$ ) in a grey to dark blue matrix

Table 1

Classification and petrological features of the igneous units of the Sierra Norte-Ambargasta batholith

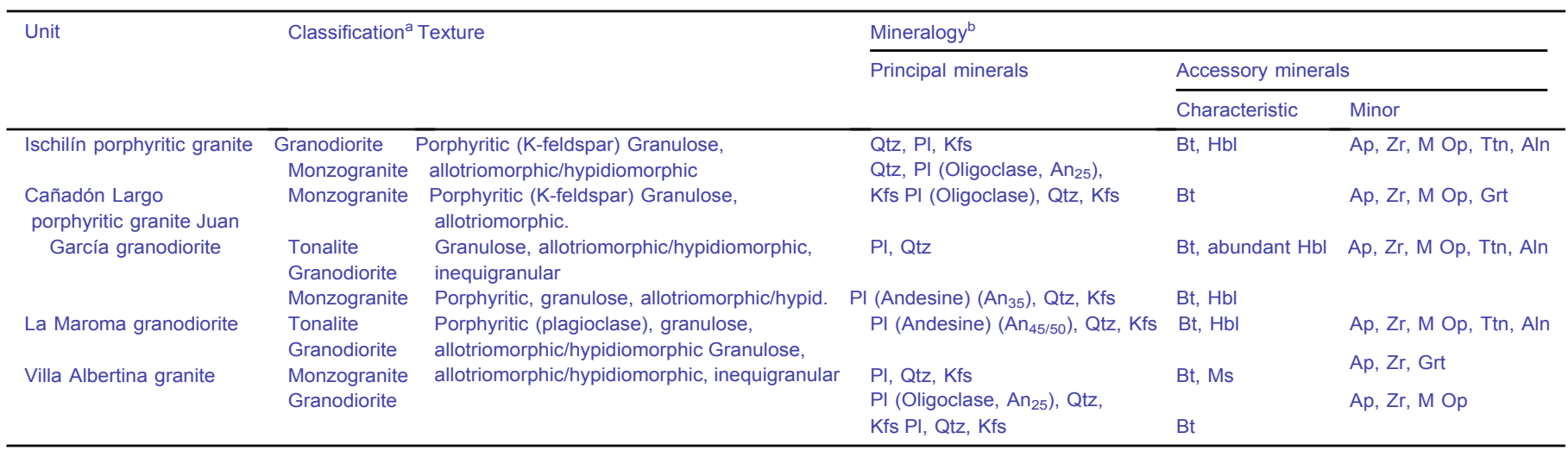

\footnotetext{
after Streckeisen (1976)

b mineral name abbreviations from Kretz (1983).
} 


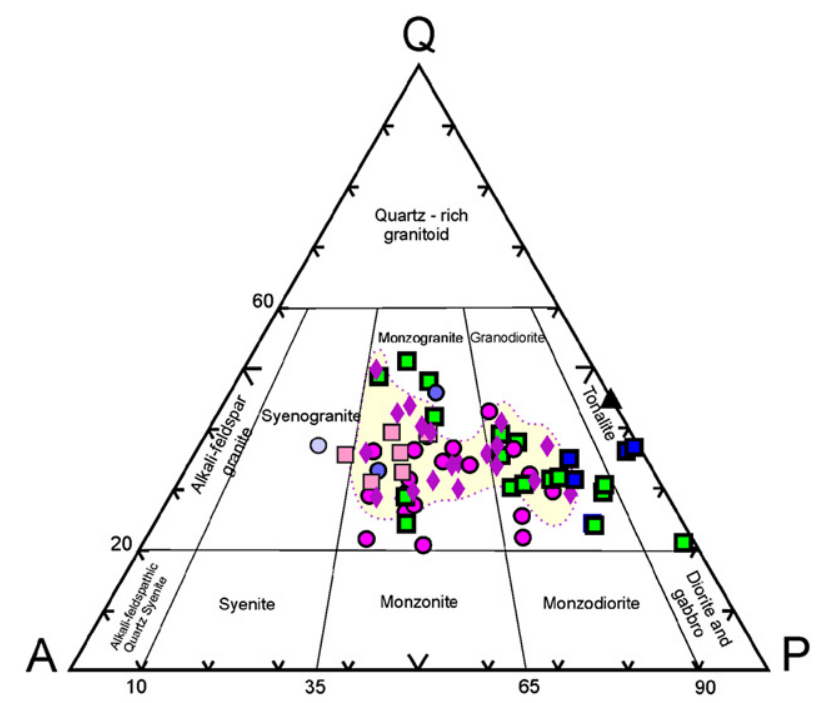

\section{DYKES: \\ Monzogranitic Pink aplite \\ O Syenogranitic dyke}

A Micro-tonalite dyke

POST-MYLONITIC UNIT:

Villa Albertina granite

\section{PRE-ISYN-MYLONITIC UNITS:}

$\square$ La Maroma granodiorite

$\square$ Juan García granodiorite

Ischilín porphyritic granite

Cañadón Largo porphyritic granite

Fig. 3. Diagram of Streckeisen (1976) showing the modal distribution of pre-/syn- and post-mylonitic units in the southern Sierra Norte-Ambargasta batholith and the associated dykes.

(Fig. 3; Table 1). Like the igneous units described above, this carries microgranular mafic enclaves, metamorphic enclaves and pink aplite bodies. Although no mylonites were found in this unit, its mineralogical and geochemical features link it with the magmatism that generated the pre-/syn-mylonitic units (see Section 6).

\subsection{Post-mylonitic units}

The Villa Albertina granite (Gordillo, 1996; lannizzotto, 2010) i s an equigranular leucocratic granite occurring as irregular outcrops in the Sierra de Ischilín. It cuts the Sauce Puncoelschilín mylonitic belt and the pre-/syn-mylonitic units as well as the metamorphic basement (Fig. 2). The main body covers an area close to $200 \mathrm{~km}^{2}$ but the principal outcrops are around the town of Villa Albertina; minor outcrops occur in eastern Sierra de Macha, with clear-cut intrusive contacts against the Ischilín porphyritic granite and the mylonites. The mapped unit includes the sub-circular leuco-cratic body of the Los Miquilos granite, which intrudes the Juan García granodiorite (Fig. 2), the Totoral granite in Sierra de Macha (Kirschbaum et al., 1997) and the equigranular biotiteemuscoviteegarnet granites of Candiani et al. (2001). This predominantly white granite lacks microgranular mafic enclaves but bears abundant mylonitic and metamorphic xenoliths. Petrographically it is leucocratic, medium- to fine-grained, and of monzogranite or granodiorite composition (Fig. 3; Table 1) with biotite, muscovite and garnet.

\subsection{Associated dykes}

Pink aplitic bodies occur throughout the Sierra NorteAmbargasta batholith (Gordillo, 1996; Lira et al., 1997; Candiani et al., 2001; Miró and Sapp, 2001). In the study area, pink aplitic bodies are widely distributed in the Sierra de Ischilín, Sierra de La Higuerita and Sierra de Macha (Figs. 2 and 3). They are dominantly tabular, with variable direction and thickness (up to $1.5 \mathrm{~m}$ ). Some show evidence of being syn-magmatic with the Ischilín porphyritic granite, such as their sinuous form; others exhibit small joints and microfaults with centimetric displacements. They show geochemical similarities to the Villa Albertina granite (see Section 6), but they have not been independently dated.

Micro-tonalite dykes appear as scarce black fine-grained rocks in small lensoid bodies that intrude the Ischilín porphyritic granite.
Similar dykes have been described from this batholith (Miró and Sapp, 2001; Koukharsky et al., 2003) and were dated at $512.6 \pm 3.5$ Ma (UePb SHRIMP) by Leal et al. (2003). Dacite, andesite and andesite-basaltic dykes have been described in the northern part of the batholith by Koukharsky et al. (2003), who obtained a KeAr whole-rock age of $514 \pm 14 \mathrm{Ma}$. In the study area, micro-tonalite dykes were found in the Copacabana locality of Sierra de La Higuerita, intruding the Ischilín porphyritic granite with a cross-cutting to transitional contact, and in Sierra de Macha, where they are interpreted as coetaneous with the host rock (lannizzotto, 2010) and thus assigned to the pre-/syn-mylonitic group.

In the northern sector of Sierra de Macha, a grey syenogranite dyke intrudes the Ischilín porphyritic granite, but the contacts are covered by modern deposit (lannizzotto, 2010) ( Fig. 3). This is grey and fine- to medium-grained, composed of quartz, plagioclase, Kfeldspar, biotite, apatite, zircon, opaque minerals and allanite. In composition it resembles the Villa Albertina granite (see Section 6), from which it is distinguished by the presence of allanite. Two syenogranitic dykes were found in Sierra de Macha. There are no geochronological data from these dykes.

\subsection{Enclaves and xenoliths}

The granites that form the southern Sierra Norte-Ambargasta batholith bear abundant enclaves, varying in size and lithology. Field observations indicate three types: 1) metamorphic basement xenoliths occur in the Cañadón Largo porphyritic granite, Ischilín porphyritic granite, Juan García granodiorite, La Maroma granodiorite and Villa Albertina granite; 2) microgranular mafic enclaves (Didier and Barbarin, 1991; Vernon, 1984) are found in the Cañadón Largo porphyritic granite, Ischilín porphyritic granite, Juan García granodiorite and La Maroma granodiorite. They have rounded, ovoid and irregular shapes, are black to dark grey and fine grained, with major axes coincident with the magmatic foliation. They are variable in size, but mostly less than $15 \mathrm{~cm}$ long, with hol-ocrystalline, inequigranular and allotriomorphic textures. They are composed of plagioclase, quartz, biotite, hornblende, titanite, allanite, apatite and opaque minerals; 3 ) mylonite xenoliths are observed in the Villa Albertina granite (Gordillo, 1996; lannizzotto et al., 2011). They show sharp contacts and a variety of shapes: elongated, elliptical, with curvilinear to angular borders. 


\subsection{The Sauce Puncoelschilín mylonitic belt}

This affects the Cañadón Largo porphyritic granite, the Ischilín porphyritic granite and the Juan García granodiorite (Gordillo, 1996; Gordillo et al.,1997; Martino, 2003; lannizzotto, 2010). No mylonites were observed within the La Maroma granodiorite. In the Ischilín area, where the NNEeSSW belt is c. $15 \mathrm{~km}$ long and c. $1 \mathrm{~km}$ wide, Gordillo (1996) distinguished northern and southern sectors: in the southern sector foliation is $350^{\circ} /\left(10 \mathrm{e} 40^{\circ}\right) \mathrm{W}$, while in the northern sector it is $320^{\circ} / 40^{\circ} \mathrm{W}$ with a lineation trending $N 190^{\circ} / 10^{\circ}$. In the Sauce Punco area, north of Sierra de Ischilín (Fig. 1), Martino et al. (1999a, b) described a $2 \mathrm{e} 7 \mathrm{~km}$ wide belt extending for $50 \mathrm{~km}$ in a general $\mathrm{N} 35^{\circ}$ direction, composed of protomylonites, mylonites and ultramylonites, and derived from granitic protoliths. A dextral sense of movement was inferred, using $\mathrm{S}$ and $\mathrm{d}$ porphyroclasts, S-C fabrics and extensional crenulation cleavages as kinematics indi-cators (Martino, 2003). A common characteristic along the entire length of the belt is the development of deformed K-feldspar por-phyroclasts and bands of quartz-rich and K-feldspar-rich layers.

In the study area (Fig. 1), the belt is defined by foliated protomylonites and mylonites, trending $\mathrm{N} 330^{\circ}$ to $\mathrm{N} 50^{\circ}$, dipping $35^{\circ} \mathrm{NW}$ to subvertical, with very well developed K-feldspar porphyroclasts. Lineations trending $\mathrm{N} 220^{\circ}$ to $\mathrm{N} 190^{\circ}$ and dipping $40 \mathrm{e} 10^{\circ}$ are defined by the orientation of biotite and quartz; S-C fabrics and $S$ porphyroblasts indicate dextral movement. On the IschilínAvellaneda road across Cerro Los Sauces there is also an outcrop of a cataclasite.

Although the mylonites are compelling evidence of strong deformation in the area, there are large sectors without mylonites, but generally with more subtle evidence of deformation, such as magmatic foliation and lineation, and orientation of phenocrysts and elongation of the mafic enclaves parallel or subparallel to the mylonitic foliation. This shows that most of the granites were emplaced during a transition from magmatic to solid-state dextral deformation. In the Cañadón Largo porphyritic granite a mylonitic zone with an orientation $346^{\circ} / 46^{\circ} \mathrm{E}$ alternates with a subparallel zone of elongated mafic igneous enclaves and K-feldspar phenocrysts. Large outcrops of the Juan García granodiorite have mafic enclaves, elongated $N 310 \mathrm{e} 330^{\circ}$ coincident with the magmatic foliation. In the Ischilín porphyritic granite there are sectors with a magmatic foliation defined by orientation of biotite sheets, feldspar phenocrysts, xenoliths of metamorphic rocks and microgranular mafic enclaves subparallel to the mylonitic foliation.

The Villa Albertina granite is the only unit that does not show any evidence of ductile deformation and was not affected by the mylonitic deformation. This unit intruded the pre-/syn-mylonitic plutons and the host basement rocks of the La Falda metamorphic complex. Outside the study area the post-mylonitic granites are volumetrically subordinate to the abundant pre-/syn-deforma-tional units spatially associated with the mylonitic zones, which define the NNE alignment of the batholith (Figs. 1 and 2).

\section{Analytical methods}

\subsection{Geochemistry}

Whole-rock samples of the pre-/syn-mylonitic and postmylonitic units and dykes were analysed for major and trace element compositions (including REE), by ICP and ICP-MS in ACT-LABS laboratories, Canada. Data are presented in Table 2.

\subsection{Geochronology}

Zircons were separated and analysed for UeThePb isotopic compositions using SHRIMP RG at The Australian National
University, Canberra, Australia, following the methods of Williams (1998, and references therein). Data were reduced using the SQUID Excel macro of Ludwig (2001); plots and calculations were performed with ISOPLOT/Ex (Ludwig, 2003). Data are listed in Tables 3 and 4 . Analyses that were $>10 \%$ discordant or with $>2.5 \%{ }^{206} \mathrm{~Pb}$ of common origin were not included in calculations.

\subsection{Sr and $\mathrm{Nd}$ isotope analyses}

$\mathrm{RbeSr}$ and SmeNd analyses of fourteen representative samples were carried out at the Geochronology and Isotope Geochemistry Centre, Complutense University (Madrid, Spain) using a VG SECTOR 54 automated multicollector mass spectrometer. Concentrations of $\mathrm{Rb}$ and $\mathrm{Sr}$ were determined by XRF spectrometry at the X-Ray Diffraction Centre of Complutense University. $\mathrm{Sm}$ and $\mathrm{Nd}$ were determined by isotope dilution using spikes enriched in ${ }^{149} \mathrm{Sm}$ and ${ }^{150} \mathrm{Nd}$. $\mathrm{Sr}$ and REE were separated using Bio-Rad AG50*12 cation exchange resin; Sm and Nd were further separated from the REE group using Bio-beads coated with $10 \%$ HDEHP. See Table 5 for further details.

\section{Geochemistry}

The plutons of the southern Sierra Norte-Ambargasta batholith show a range of silica contents between $62 \%$ and $78 \%$ (Table 2 ), the monzogranitic pink aplites being the most siliceous unit, and the tonalites of La Maroma granodiorite and the mafic dykes the most basic. All the units are subalkaline in the TAS diagram (lannizzotto, 2010).

The samples of Ischilín porphyritic granite and Juan García granodiorite cannot be separated in the terms of major element composition (Figs. 3 and 4), coinciding with their close association in the field and the absence of clear contacts between them. They represent the main magmatic episode in this area and can be compared to the GM series of Lira et al. (1997) in the central part of the batholith. On the other hand, the Villa Albertina granite is more leucocratic, depleted in HREE and slightly enriched in LIL elements in comparison with the pre-/syn-mylonitic units (Figs. 5 and 6). Overall, the decrease in $\mathrm{TiO}_{2}$ and $\mathrm{P}_{2} \mathrm{O}_{5}$ (Table 2) with increasing $\mathrm{SiO}_{2}$ matches the decrease in modal titanite and apatite in the La Mar-oma granodiorite, the Ischilín porphyritic granite and Juan García granodiorite.

In the diagram of Frost et al. (2001), the pre-/syn-mylonitic plutons fall in the fields of calcalkalic to calcic and magnesian magmas (Fig. 4), typical of granitic rocks related to an active continental margin. A transition from metaluminous to weakly peraluminous composition is observed in these units, whereas the Villa Albertina granite is peraluminous and more similar to granites in the central and northern part of the batholith (Bonalumi, 1988; Lira et al., 1997) and the Pampean granites of the Sierra de Córdoba (Rapela et al., 2008). REE patterns of the pre-/syn-mylonitic granites conform to the calc-alkaline series (Fig. 5), while the chondritenormalized spider diagram shows the typical $\mathrm{Nb}$ and Ta depletion of magmas formed in subduction zones (Fig. 6). It should be pointed out that despite the Villa Albertina granite showing marked differences in field relations, lack of deformation and lighter colour index, its REE patterns are not distinctive.

\section{Geochronology}

\subsection{Background}

Earlier geochronological studies of the Sierra NorteAmbargasta batholith include those of Castellote (1982), 
Geochemical analyses of samples, granitoids and country metamorphic rocks from the Sierra Norte-Ambargasta batholith. ACTLABS Laboratories, Canada.

\begin{tabular}{|c|c|c|c|c|c|c|c|c|c|c|c|c|c|c|c|c|c|c|}
\hline \multirow{2}{*}{$\begin{array}{l}\text { Unit } \\
\text { Sample } \\
\text { (NF Is) }\end{array}$} & \multicolumn{2}{|c|}{ Met. basement } & \multicolumn{2}{|c|}{ La Maroma G. } & \multicolumn{4}{|c|}{ Ischilín porphiritic granite } & \multicolumn{2}{|c|}{ Juan García G. } & \multicolumn{5}{|c|}{ Villa Albertina granite } & \multirow{2}{*}{$\frac{\text { Aplite }}{86}$} & \multirow{2}{*}{$\frac{\text { M. Dyke }}{132}$} & \multirow{2}{*}{$\frac{\text { S. Dyke }}{123}$} \\
\hline & 115 & 138 & 128 & 134 & 114 & 117 & 119 & 125 & 23 & 149 & 8 & 13 & 20 & 62 & 108 & & & \\
\hline (wt \%) & & & & & & & & & & & & & & & & & & \\
\hline $\mathrm{SiO}_{2}$ & 67.34 & 69.3 & 60.75 & 67.18 & 65.8 & 66.31 & 68.07 & 71.73 & 66.76 & 66.26 & 71.43 & 71.66 & 73.75 & 72.72 & 73.58 & 77.26 & 62.55 & 71.03 \\
\hline $\mathrm{TiO}_{2}$ & 0.61 & 0.77 & 1.04 & 0.6 & 0.72 & 0.74 & 0.48 & 0.24 & 0.47 & 0.53 & 0.3 & 0.17 & 0.13 & 0.23 & 0.2 & 0.07 & 0.49 & 0.31 \\
\hline $\mathrm{Fe}_{2} \mathrm{O}_{3 \mathrm{t}}$ & 4.93 & 5.65 & 7.26 & 4.37 & 5.84 & 5.58 & 4.33 & 2.77 & 3.98 & 4.57 & 2.58 & 1.73 & 1.71 & 2.3 & 1.99 & 1.41 & 4.24 & 2.46 \\
\hline $\mathrm{MnO}$ & 0.09 & 0.1 & 0.12 & 0.11 & 0.1 & 0.09 & 0.08 & 0.07 & 0.08 & 0.03 & 0.03 & 0.03 & 0.03 & 0.06 & 0.06 & 0.02 & 0.07 & 0.04 \\
\hline $\mathrm{MgO}$ & 2.19 & 2.12 & 3 & 1.41 & 1.14 & 1.22 & 1.08 & 0.38 & 1.18 & 1.91 & 0.72 & 0.41 & 0.33 & 0.58 & 0.46 & 0.04 & 2.5 & 0.64 \\
\hline $\mathrm{CaO}$ & 2.03 & 2.33 & 5.49 & 3.39 & 3 & 2.54 & 3.35 & 1.33 & 3.41 & 3.89 & 2.68 & 1.93 & 1.46 & 2.74 & 1.64 & 1.01 & 5.12 & 2.63 \\
\hline $\mathrm{Na}_{2} \mathrm{O}$ & 3.16 & 2.01 & 3.48 & 3.58 & 3.41 & 3.35 & 2.98 & 3.5 & 3.16 & 3.3 & 3.37 & 3 & 3.19 & 3.66 & 3 & 2.64 & 3.85 & 3.9 \\
\hline $\mathrm{K}_{2} \mathrm{O}$ & 3.8 & 3.19 & 2.42 & 3.27 & 3.79 & 3.74 & 3.56 & 4.6 & 4.06 & 3.17 & 3.66 & 4.76 & 4.55 & 2.13 & 4.75 & 4.99 & 1.89 & 3.11 \\
\hline $\mathrm{P}_{2} \mathrm{O}_{5}$ & 0.16 & 0.17 & 0.51 & 0.22 & 0.28 & 0.29 & 0.13 & 0.1 & 0.13 & 0.15 & 0.12 & 0.06 & 0.05 & 0.07 & 0.06 & 0.02 & 0.11 & 0.1 \\
\hline LOI & 0.69 & 0.73 & 0.59 & 0.59 & 0.61 & 0.66 & 0.51 & 1.02 & 0.73 & 0.89 & 0.49 & 0.42 & 0.58 & 0.64 & 0.54 & 0.33 & 1.88 & 0.37 \\
\hline $\begin{array}{l}\text { Total } \\
\text { ppm }\end{array}$ & 99.98 & 98.83 & 100.5 & 99.91 & 100.2 & 99.83 & 99.72 & 99.79 & 99.73 & 100.7 & 100.8 & 98.52 & 99.77 & 100.1 & 100.1 & 99.67 & 100.1 & 99.69 \\
\hline Cs & 9.2 & 8.2 & 8.4 & 10.5 & 9 & 8.4 & 11.4 & 21.5 & 19 & 10.4 & 5.5 & 4.9 & 4 & 9.2 & 19.8 & 0.7 & 2.9 & 6.4 \\
\hline $\mathrm{Rb}$ & 144 & 163 & 93 & 136 & 153 & 179 & 124 & 238 & 170 & 134 & 108 & 116 & 123 & 135 & 187 & 74 & 59 & 97 \\
\hline $\mathrm{Sr}$ & 268 & 154 & 281 & 160 & 184 & 148 & 163 & 77 & 168 & 230 & 301 & 227 & 217 & 311 & 106 & 109 & 427 & 322 \\
\hline $\mathrm{Ba}$ & 719 & 573 & 478 & 456 & 503 & 442 & 637 & 639 & 504 & 466 & 762 & 1181 & 1109 & 767 & 497 & 1042 & 383 & \\
\hline La & 41.3 & 66.7 & 46.1 & 39.6 & 55 & 53.4 & 35.3 & 44.8 & 24.6 & 27.8 & 30.4 & 33.3 & 30.8 & 36.2 & 26.3 & 19.7 & 16.6 & 40.6 \\
\hline $\mathrm{Ce}$ & 78.6 & 128 & 91.7 & 77.1 & 107 & 108 & 65.4 & 89.4 & 52.6 & 55.3 & 57.1 & 61.9 & 60.6 & 65.8 & 49.6 & 44.1 & 31.1 & 87.8 \\
\hline $\mathrm{Pr}$ & 8.55 & 14.4 & 10.6 & 8.66 & 12.2 & 12.5 & 7.14 & 9.87 & 6.05 & 5.76 & 6.1 & 6.74 & 6.51 & 6.57 & 5.83 & 5.17 & 3.42 & 7.11 \\
\hline $\mathrm{Nd}$ & 29.9 & 47.9 & 38.2 & 33.9 & 41.7 & 48.9 & 24.8 & 37.8 & 22.5 & 21.7 & 20.9 & 22.4 & 22.2 & 21 & 22.2 & 21.9 & 12 & 20.8 \\
\hline $\mathrm{Sm}$ & 6.53 & 9.95 & 8.5 & 7.87 & 9.25 & 11.3 & 5.44 & 8.79 & 4.73 & 5.06 & 4.39 & 4.71 & 4.14 & 4.22 & 4.93 & 5.71 & 2.59 & 3.83 \\
\hline Eu & 1.38 & 1.22 & 2.04 & 1.5 & 2.08 & 1.87 & 1.15 & 1.55 & 1.06 & 1.01 & 1.05 & 1.16 & 0.92 & 0.8 & 0.83 & 0.96 & 0.74 & 1.01 \\
\hline $\mathrm{Gd}$ & 5.1 & 7.07 & 6.84 & 7.24 & 7.09 & 9.27 & 4.53 & 8.2 & 4.32 & 4.38 & 3.18 & 3.75 & 3.62 & 3.08 & 3.93 & 4.36 & 1.8 & 2.22 \\
\hline $\mathrm{Tb}$ & 0.82 & 0.83 & 1.06 & 1.36 & 1.04 & 1.51 & 0.75 & 1.46 & 0.74 & 0.74 & 0.53 & 0.52 & 0.61 & 0.53 & 0.66 & 0.57 & 0.25 & 0.33 \\
\hline Dy & 4.85 & 3.79 & 6.03 & 8.5 & 5.8 & 9.01 & 4.53 & 9.42 & 4.29 & 4.58 & 3.06 & 2.87 & 3.52 & 3.11 & 4.11 & 2.6 & 1.43 & 1.87 \\
\hline Ho & 1.01 & 0.61 & 1.25 & 1.81 & 1.15 & 1.81 & 0.96 & 2.03 & 0.85 & 0.95 & 0.62 & 0.58 & 0.69 & 0.63 & 0.87 & 0.42 & 0.28 & 0.4 \\
\hline $\mathrm{Er}$ & 3.11 & 1.63 & 3.85 & 5.66 & 3.39 & 5.29 & 3.15 & 6.41 & 2.59 & 2.83 & 1.91 & 1.73 & 2.09 & 2.02 & 2.84 & 1.03 & 0.85 & 1.37 \\
\hline $\mathrm{Tm}$ & 0.46 & 0.238 & 0.559 & 0.834 & 0.473 & 0.743 & 0.496 & 0.98 & 0.41 & 0.404 & 0.291 & 0.254 & 0.35 & 0.333 & 0.441 & 0.132 & 0.123 & 0.228 \\
\hline $\mathrm{Yb}$ & 2.82 & 1.53 & 3.39 & 4.85 & 2.79 & 4.29 & 3.25 & 6.13 & 2.66 & 2.46 & 1.87 & 1.6 & 2.34 & 2.27 & 2.8 & 0.78 & 0.74 & 1.55 \\
\hline Lu & 0.402 & 0.22 & 0.491 & 0.64 & 0.4 & 0.597 & 0.489 & 0.872 & 0.399 & 0.338 & 0.267 & 0.235 & 0.355 & 0.33 & 0.393 & 0.111 & 0.103 & 0.272 \\
\hline U & 2.87 & 2.15 & 2.38 & 3.46 & 3.06 & 2.39 & 4.63 & 5.67 & 2.84 & 2.64 & 2.02 & 1.9 & 2.33 & 2.26 & 5.07 & 1.78 & 1.69 & 3.75 \\
\hline Th & 12.9 & 24.4 & 8.42 & 11.5 & 17.5 & 22.8 & 10.9 & 21.8 & 13.4 & 14.3 & 8.83 & 14 & 12.7 & 15.8 & 16.9 & 14.7 & 4.92 & 10.6 \\
\hline $\mathrm{Nb}$ & 12.1 & 12.5 & 16.5 & 16.3 & 19.2 & 25.7 & 10.2 & 20.2 & 12.1 & 8.8 & 9.7 & 6.8 & 9.2 & 12.5 & 10.9 & 1.7 & 3.2 & 6.5 \\
\hline $\mathrm{Zr}$ & 183 & 317 & 328 & 250 & 335 & 312 & 168 & 282 & 146 & 179 & 141 & 116 & 70 & 143 & 111 & 106 & 95 & 216 \\
\hline $\mathrm{Hf}$ & 5 & 8.8 & 7.6 & 6.4 & 8.6 & 8.5 & 4.7 & 8.2 & 4.4 & 4.9 & 3.9 & 3.1 & 2.3 & 4 & 3.6 & 4.7 & 2.7 & 5.2 \\
\hline $\mathrm{Ta}$ & 1.4 & 1.43 & 1.24 & 3.31 & 1.71 & 2.13 & 1.49 & 2.91 & 1.54 & 1.04 & 1.37 & 0.86 & 0.91 & 2.76 & 1.98 & 0.07 & 0.27 & 0.63 \\
\hline $\mathrm{Sc}$ & 11 & 14 & 16 & 10 & 8 & 9 & 9 & 8 & 9 & 19 & 5 & 5 & 5 & 4 & 5 & 2 & 11 & 4 \\
\hline Ga & 17 & 16 & 18 & 17 & 19 & 21 & 15 & 19 & 17 & 17 & 17 & 13 & 14 & 15 & 13 & 11 & 19 & 15 \\
\hline $\mathrm{Ni}$ & 40 & 30 & 30 & $<20$ & $<20$ & $<20$ & $<20$ & $<20$ & $<20$ & $<20$ & $<20$ & $<20$ & $<20$ & $<20$ & $<20$ & $<20$ & $<20$ & $<20$ \\
\hline Co & 11 & 12 & 15 & 8 & 8 & & 7 & 2 & 7 & 10 & 3 & 2 & 1 & 2 & 3 & $<1$ & 1 & 03 \\
\hline $\mathrm{Cr}$ & 80 & 80 & 40 & $<20$ & 30 & $<20$ & $<20$ & 40 & $<20$ & 20 & $<20$ & 30 & 20 & 30 & $<20$ & 50 & 40 & 30 \\
\hline $\mathrm{Pb}$ & 27 & 15 & 11 & 17 & 12 & 20 & 24 & 21 & 42 & 16 & 26 & 29 & 28 & 22 & 38 & 11 & 14 & 19 \\
\hline $\mathrm{Ag}$ & $<0.5$ & $<0.5$ & 0.6 & $<0.5$ & 0.8 & $<0.5$ & $<0.5$ & $<0.5$ & $<0.5$ & $<0.5$ & $<0.5$ & $<0.5$ & $<0.5$ & $<0.5$ & $<0.5$ & 0.7 & $<0.5$ & $<0.5$ \\
\hline $\mathrm{Cu}$ & 20 & $<10$ & 50 & 30 & 80 & 30 & $<10$ & 10 & 10 & 20 & $<10$ & $<10$ & 10 & $<10$ & $<10$ & 20 & 40 & $<10$ \\
\hline V & 72 & 87 & 121 & 61 & 44 & 47 & 52 & 22 & 59 & 83 & 29 & 19 & 13 & 19 & 22 & $<5$ & 100 & 30 \\
\hline $\mathrm{Zn}$ & 90 & 100 & 110 & 80 & 90 & 110 & 70 & 60 & 60 & 60 & 60 & 30 & 60 & 50 & 80 & $<30$ & 60 & 40 \\
\hline B & e 3 & 2 & 3 & 4 & 3 & 2 & 3 & 8 & 3 & 4 & 3 & 2 & 2 & 4 & 4 & 1 & 3 & 3 \\
\hline $\mathrm{Ge}$ & 1.4 & 1.1 & 1.2 & 1.3 & 1.2 & 1.2 & 1.3 & 1.5 & 1.5 & 1.2 & 1.2 & 1 & 1.2 & 1 & 1.5 & 1 & 0.8 & 0.9 \\
\hline Y & 29.1 & 16.9 & 35.7 & 51.8 & 31.8 & 52.1 & 29.3 & 58.3 & 27.9 & 25.7 & 19.3 & 16.4 & 22.1 & 20.2 & 26.2 & 9.4 & 8.7 & 12.8 \\
\hline Mo & $<2$ & $<2$ & $<2$ & $<2$ & $<2$ & $<2$ & $<2$ & $<2$ & $<2$ & $<2$ & $<2$ & $<2$ & $<2$ & $<2$ & $<2$ & $<2$ & $<2$ & $<2$ \\
\hline s & n3 & 6 & 2 & 4 & 3 & 6 & 2 & 8 & 2 & 2 & 1 & 1 & $<1$ & 5 & 3 & $<1$ & $<1$ & $<1$ \\
\hline $\mathrm{Sb}$ & 3.7 & 1.6 & 4.8 & 3.2 & 1.7 & 1.4 & 5.1 & 1.6 & 0.3 & 1.8 & 3 & 1.8 & $<0.2$ & 2.9 & 4.5 & 3.3 & $<0.2$ & 1.9 \\
\hline
\end{tabular}


Table 2 (continued)

\begin{tabular}{|c|c|c|c|c|c|c|c|c|c|c|c|c|c|c|c|c|c|c|}
\hline \multirow{2}{*}{$\begin{array}{l}\text { Unit } \\
\text { Sample } \\
\text { (NF Is) }\end{array}$} & \multicolumn{2}{|c|}{ Met. basement } & \multicolumn{2}{|c|}{ La Maroma G. } & \multicolumn{4}{|c|}{ Ischilín porphiritic granite } & \multicolumn{2}{|c|}{ Juan García G. } & \multicolumn{5}{|c|}{ Villa Albertina granite } & \multirow{2}{*}{$\frac{\text { Aplite }}{86}$} & \multirow{2}{*}{$\frac{\text { M. Dyke }}{132}$} & \multirow{2}{*}{$\frac{\text { S. Dyke }}{123}$} \\
\hline & 115 & 138 & 128 & 134 & 114 & 117 & 119 & 125 & 23 & 149 & 8 & 13 & 20 & 62 & 108 & & & \\
\hline $\mathbf{w}$ & 1.4 & 0.8 & 0.7 & $<0.5$ & 0.6 & 0.8 & 1.1 & 3.7 & 0.6 & 0.8 & 0.8 & $<0.5$ & $<0.5$ & 1 & 0.5 & $<0.5$ & $<0.5$ & $<0.5$ \\
\hline TI & 1.14 & 1.29 & 0.76 & 1.09 & 1.1 & 1.38 & 0.95 & 1.45 & 1 & 1 & 0.82 & 0.87 & 0.58 & 1.18 & 1.47 & 0.48 & 0.41 & 0.75 \\
\hline $\mathrm{Bi}$ & 0.3 & $<0.1$ & 0.5 & 0.6 & 0.4 & 0.4 & 0.6 & 1.3 & 0.7 & 0.3 & 0.6 & 0.2 & $<0.1$ & 0.3 & 0.3 & $<0.1$ & 0.1 & 0.4 \\
\hline In & $<0.1$ & $<0.1$ & $<0.1$ & $<0.1$ & $<0.1$ & $<0.1$ & $<0.1$ & $<0.1$ & $<0.1$ & $<0.1$ & $<0.1$ & $<0.1$ & $<0.1$ & $<0.1$ & $<0.1$ & $<0.1$ & $<0.1$ & $<0.1$ \\
\hline $\mathrm{Er}$ & 3.11 & 1.63 & 3.85 & 5.66 & 3.39 & 5.29 & 3.15 & 6.41 & 2.59 & 2.83 & 1.91 & 1.73 & 2.09 & 2.02 & 2.84 & 1.03 & 0.85 & 1.37 \\
\hline $\mathrm{Tm}$ & 0.46 & 0.238 & 0.559 & 0.834 & 0.473 & 0.743 & 0.496 & 0.98 & 0.41 & 0.404 & 0.291 & 0.254 & 0.35 & 0.333 & 0.441 & 0.132 & 0.123 & 0.228 \\
\hline$Y b$ & 2.82 & 1.53 & 3.39 & 4.85 & 2.79 & 4.29 & 3.25 & 6.13 & 2.66 & 2.46 & 1.87 & 1.6 & 2.34 & 2.27 & 2.8 & 0.78 & 0.74 & 1.55 \\
\hline Lu & 0.402 & 0.22 & 0.491 & 0.64 & 0.4 & 0.597 & 0.489 & 0.872 & 0.399 & 0.338 & 0.267 & 0.235 & 0.355 & 0.33 & 0.393 & 0.111 & 0.103 & 0.272 \\
\hline u & 2.87 & 2.15 & 2.38 & 3.46 & 3.06 & 2.39 & 4.63 & 5.67 & 2.84 & 2.64 & 2.02 & 1.9 & 2.33 & 2.26 & 5.07 & 1.78 & 1.69 & 3.75 \\
\hline Th & 12.9 & 24.4 & 8.42 & 11.5 & 17.5 & 22.8 & 10.9 & 21.8 & 13.4 & 14.3 & 8.83 & 14 & 12.7 & 15.8 & 16.9 & 14.7 & 4.92 & 10.6 \\
\hline Nb & 12.1 & 12.5 & 16.5 & 16.3 & 19.2 & 25.7 & 10.2 & 20.2 & 12.1 & 8.8 & 9.7 & 6.8 & 9.2 & 12.5 & 10.9 & 1.7 & 3.2 & 6.5 \\
\hline $\mathrm{Zr}$ & 183 & 317 & 328 & 250 & 335 & 312 & 168 & 282 & 146 & 179 & 141 & 116 & 70 & 143 & 111 & 106 & 95 & 216 \\
\hline $\mathrm{Hf}$ & 5 & 8.8 & 7.6 & 6.4 & 8.6 & 8.5 & 4.7 & 8.2 & 4.4 & 4.9 & 3.9 & 3.1 & 2.3 & 4 & 3.6 & 4.7 & 2.7 & 5.2 \\
\hline $\mathrm{Ta}$ & 1.4 & 1.43 & 1.24 & 3.31 & 1.71 & 2.13 & 1.49 & 2.91 & 1.54 & 1.04 & 1.37 & 0.86 & 0.91 & 2.76 & 1.98 & 0.07 & 0.27 & 0.63 \\
\hline Sc & 11 & 14 & 16 & 10 & 8 & 9 & 9 & 8 & 9 & 19 & 5 & 5 & 5 & 4 & 5 & 2 & 11 & 4 \\
\hline Ga & 17 & 16 & 18 & 17 & 19 & 21 & 15 & 19 & 17 & 17 & 17 & 13 & 14 & 15 & 13 & 11 & 19 & 15 \\
\hline $\mathrm{Ni}$ & 40 & 30 & 30 & $<20$ & $<20$ & $<20$ & $<20$ & $<20$ & $<20$ & $<20$ & $<20$ & $<20$ & $<20$ & $<20$ & $<20$ & $<20$ & $<20$ & $<20$ \\
\hline Co & 11 & 12 & 15 & 8 & 8 & & 7 & 2 & 7 & 10 & 3 & 2 & 1 & 2 & 3 & $<1$ & 1 & 03 \\
\hline $\mathrm{Cr}$ & 80 & 80 & 40 & $<20$ & 30 & $<20$ & $<20$ & 40 & $<20$ & 20 & $<20$ & 30 & 20 & 30 & $<20$ & 50 & 40 & 30 \\
\hline $\mathrm{Pb}$ & 27 & 15 & 11 & 17 & 12 & 20 & 24 & 21 & 42 & 16 & 26 & 29 & 28 & 22 & 38 & 11 & 14 & 19 \\
\hline $\mathrm{Ag}$ & $<0.5$ & $<0.5$ & 0.6 & $<0.5$ & 0.8 & $<0.5$ & $<0.5$ & $<0.5$ & $<0.5$ & $<0.5$ & $<0.5$ & $<0.5$ & $<0.5$ & $<0.5$ & $<0.5$ & 0.7 & $<0.5$ & $<0.5$ \\
\hline $\mathrm{Cu}$ & 20 & $<10$ & 50 & 30 & 80 & 30 & $<10$ & 10 & 10 & 20 & $<10$ & $<10$ & 10 & $<10$ & $<10$ & 20 & 40 & $<10$ \\
\hline v & 72 & 87 & 121 & 61 & 44 & 47 & 52 & 22 & 59 & 83 & 29 & 19 & 13 & 19 & 22 & $<5$ & 100 & 30 \\
\hline$Z n$ & 90 & 100 & 110 & 80 & 90 & 110 & 70 & 60 & 60 & 60 & 60 & 30 & 60 & 50 & 80 & $<30$ & 60 & 40 \\
\hline B & e 3 & 2 & 3 & 4 & 3 & 2 & 3 & 8 & 3 & 4 & 3 & 2 & 2 & 4 & 4 & 1 & 3 & 3 \\
\hline Ge & 1.4 & 1.1 & 1.2 & 1.3 & 1.2 & 1.2 & 1.3 & 1.5 & 1.5 & 1.2 & 1.2 & 1 & 1.2 & 1 & 1.5 & 1 & 0.8 & 0.9 \\
\hline$Y$ & 29.1 & 16.9 & 35.7 & 51.8 & 31.8 & 52.1 & 29.3 & 58.3 & 27.9 & 25.7 & 19.3 & 16.4 & 22.1 & 20.2 & 26.2 & 9.4 & 8.7 & 12.8 \\
\hline Mo & $<2$ & $<2$ & $<2$ & $<2$ & $<2$ & $<2$ & $<2$ & $<2$ & $<2$ & $<2$ & $<2$ & $<2$ & $<2$ & $<2$ & $<2$ & $<2$ & $<2$ & $<2$ \\
\hline $\mathbf{s}$ & n 3 & 6 & 2 & 4 & 3 & 6 & 2 & 8 & 2 & 2 & 1 & 1 & $<1$ & 5 & 3 & $<1$ & $<1$ & $<1$ \\
\hline Sb & 3.7 & 1.6 & 4.8 & 3.2 & 1.7 & 1.4 & 5.1 & 1.6 & 0.3 & 1.8 & 3 & 1.8 & $<0.2$ & 2.9 & 4.5 & 3.3 & $<0.2$ & 1.9 \\
\hline w & 1.4 & 0.8 & 0.7 & $<0.5$ & 0.6 & 0.8 & 1.1 & 3.7 & 0.6 & 0.8 & 0.8 & $<0.5$ & $<0.5$ & 1 & 0.5 & $<0.5$ & $<0.5$ & $<0.5$ \\
\hline TI & 1.14 & 1.29 & 0.76 & 1.09 & 1.1 & 1.38 & 0.95 & 1.45 & 1 & 1 & 0.82 & 0.87 & 0.58 & 1.18 & 1.47 & 0.48 & 0.41 & 0.75 \\
\hline $\mathrm{Bi}$ & 0.3 & $<0.1$ & 0.5 & 0.6 & 0.4 & 0.4 & 0.6 & 1.3 & 0.7 & 0.3 & 0.6 & 0.2 & $<0.1$ & 0.3 & 0.3 & $<0.1$ & 0.1 & 0.4 \\
\hline In & $<0.1$ & $<0.1$ & $<0.1$ & $<0.1$ & $<0.1$ & $<0.1$ & $<0.1$ & $<0.1$ & $<0.1$ & $<0.1$ & $<0.1$ & $<0.1$ & $<0.1$ & $<0.1$ & $<0.1$ & $<0.1$ & $<0.1$ & $<0.1$ \\
\hline
\end{tabular}

Analyses carried out at ACTLABS, Canada. 
Table 3

UePb SHRIMP analyses of zircon samples from the Sierra Norte-Ambargasta batholith.

\begin{tabular}{|c|c|c|c|c|c|c|c|c|c|c|c|c|c|c|}
\hline \multirow[t]{2}{*}{ Grain. spot } & \multirow[t]{2}{*}{$\mathrm{U}(\mathrm{ppm})$} & \multirow[t]{2}{*}{ Th (ppm) } & \multirow[t]{2}{*}{$\mathrm{Th} / \mathrm{U}$} & \multirow{2}{*}{$\begin{array}{r}{ }^{206} \mathrm{~Pb}^{*} \\
(\mathrm{ppm})\end{array}$} & \multirow[t]{2}{*}{${ }^{204} \mathrm{~Pb} /{ }^{206} \mathrm{~Pb}$} & \multirow[t]{2}{*}{ f206\% } & \multicolumn{4}{|c|}{ Measured ratios } & \multirow{2}{*}{\multicolumn{2}{|c|}{$\frac{\text { Radiogenic ratios }}{{ }^{206} \mathrm{~Pb} /{ }^{238} \mathrm{U} \pm}$}} & \multirow{2}{*}{\multicolumn{2}{|c|}{$\begin{array}{l}\text { Age (Ma) } \\
{ }^{206} \mathrm{~Pb} /{ }^{238} \mathrm{U} \pm\end{array}$}} \\
\hline & & & & & & & \multicolumn{2}{|c|}{${ }^{238} \mathrm{U} /{ }^{206} \mathrm{~Pb} \pm$} & \multicolumn{2}{|c|}{${ }^{207} \mathrm{~Pb} /{ }^{206} \mathrm{~Pb} \pm$} & & & & \\
\hline \multicolumn{15}{|c|}{ Sample NF-Is-23 Juan Garcia granodiorite } \\
\hline 1.1 & 422 & 201 & 0.48 & 32.0 & 0.000031 & 0.08 & 11.334 & 0.121 & 0.0590 & 0.0005 & 0.0882 & 0.0010 & 544.7 & 5.7 \\
\hline $2.1^{*}$ & 844 & 269 & 0.32 & 59.9 & 0.001009 & 2.06 & 12.107 & 0.271 & 0.0740 & 0.0013 & 0.0809 & 0.0018 & 501.4 & 11.0 \\
\hline 3.1 & 225 & 71 & 0.32 & 16.4 & 0.000450 & 1.16 & 11.747 & 0.133 & 0.0672 & 0.0008 & 0.0841 & 0.0010 & 520.8 & 5.8 \\
\hline 4.1 & 465 & 138 & 0.30 & 35.0 & 0.000300 & 0.46 & 11.398 & 0.121 & 0.0620 & 0.0005 & 0.0873 & 0.0009 & 539.7 & 5.6 \\
\hline 5.1 & 669 & 148 & 0.22 & 50.3 & 0.000071 & 0.14 & 11.429 & 0.120 & 0.0594 & 0.0004 & 0.0874 & 0.0009 & 540.0 & 5.5 \\
\hline $6.1^{*}$ & 735 & 240 & 0.33 & 59.0 & 0.000115 & 0.03 & 10.710 & 0.251 & 0.0595 & 0.0004 & 0.0933 & 0.0022 & 575.2 & 13.1 \\
\hline 7.1 & 594 & 260 & 0.44 & 44.9 & $\mathrm{e}$ & 0.07 & 11.369 & 0.121 & 0.0589 & 0.0004 & 0.0879 & 0.0010 & 543.1 & 5.7 \\
\hline 8.1 & 421 & 135 & 0.32 & 31.9 & 0.000013 & 0.20 & 11.352 & 0.122 & 0.0600 & 0.0005 & 0.0879 & 0.0010 & 543.2 & 5.7 \\
\hline 9.1 & 363 & 171 & 0.47 & 27.3 & 0.000011 & 0.17 & 11.441 & 0.124 & 0.0596 & 0.0005 & 0.0873 & 0.0010 & 539.3 & 5.7 \\
\hline $10.1^{*}$ & 646 & 273 & 0.42 & 50.4 & 0.006443 & 13.30 & 11.021 & 0.130 & 0.1650 & 0.0076 & 0.0787 & 0.0013 & 488.2 & 7.7 \\
\hline 11.1 & 385 & 136 & 0.35 & 28.3 & 0.000015 & 0.16 & 11.688 & 0.128 & 0.0592 & 0.0006 & 0.0854 & 0.0010 & 528.4 & 5.7 \\
\hline 12.1 & 210 & 99 & 0.47 & 15.6 & 0.000102 & $<0.01$ & 11.552 & 0.135 & 0.0578 & 0.0008 & 0.0866 & 0.0010 & 535.4 & 6.1 \\
\hline 13.1 & 1183 & 372 & 0.31 & 89.7 & 0.000143 & 0.30 & 11.321 & 0.117 & 0.0608 & 0.0003 & 0.0881 & 0.0009 & 544.1 & 5.5 \\
\hline 14.1 & 103 & 60 & 0.58 & 7.8 & 0.000236 & 0.49 & 11.415 & 0.149 & 0.0622 & 0.0011 & 0.0872 & 0.0012 & 538.8 & 6.9 \\
\hline 14.2 & 676 & 182 & 0.27 & 50.8 & 0.000024 & 0.05 & 11.432 & 0.121 & 0.0587 & 0.0004 & 0.0874 & 0.0009 & 540.3 & 5.6 \\
\hline 15.1 & 418 & 66 & 0.16 & 31.1 & $\mathrm{e}$ & $<0.01$ & 11.543 & 0.126 & 0.0581 & 0.0006 & 0.0866 & 0.0010 & 535.7 & 5.7 \\
\hline 16.1 & 739 & 192 & 0.26 & 55.0 & 0.000015 & 0.12 & 11.534 & 0.122 & 0.0591 & 0.0004 & 0.0866 & 0.0009 & 535.4 & 5.5 \\
\hline 17.1 & 617 & 154 & 0.25 & 45.9 & $\mathrm{e}$ & 0.10 & 11.535 & 0.123 & 0.0590 & 0.0005 & 0.0866 & 0.0009 & 535.4 & 5.6 \\
\hline $18.1^{*}$ & 702 & 157 & 0.22 & 49.9 & 0.001152 & 2.25 & 12.100 & 0.128 & 0.0755 & 0.0019 & 0.0808 & 0.0009 & 500.8 & 5.3 \\
\hline 19.1 & 276 & 79 & 0.29 & 20.3 & 0.000352 & 0.29 & 11.648 & 0.150 & 0.0603 & 0.0007 & 0.0856 & 0.0011 & 529.5 & 6.7 \\
\hline $20.1^{*}$ & 1115 & 663 & 0.59 & 94.2 & 0.000039 & $<0.01$ & 10.176 & 0.107 & 0.0592 & 0.0003 & 0.0984 & 0.0011 & 604.9 & 6.2 \\
\hline \multicolumn{15}{|c|}{ Sample NF-Is-20 Villa Albertina granite } \\
\hline $1.1^{*}$ & 723 & 178 & 0.25 & 48.6 & 0.000587 & 1.28 & 12.784 & 0.136 & 0.0671 & 0.0005 & 0.0772 & 0.0008 & 479.5 & 5.0 \\
\hline $2.1^{*}$ & 6655 & 1690 & 0.25 & 550.9 & 0.001062 & 1.64 & 10.380 & 0.109 & 0.0729 & 0.0008 & 0.0948 & 0.0010 & 583.6 & 6.0 \\
\hline 3.1 & 1387 & 36 & 0.03 & 104.2 & 0.000073 & 0.12 & 11.430 & 0.118 & 0.0592 & 0.0003 & 0.0874 & 0.0009 & 540.1 & 5.4 \\
\hline $4.1^{*}$ & 246 & 75 & 0.30 & 18.9 & 0.000019 & 0.12 & 11.164 & 0.131 & 0.0596 & 0.0008 & 0.0895 & 0.0011 & 552.4 & 6.3 \\
\hline $5.1^{*}$ & 323 & 100 & 0.31 & 24.6 & 0.000146 & 0.15 & 11.276 & 0.128 & 0.0597 & 0.0007 & 0.0885 & 0.0010 & 546.9 & 6.0 \\
\hline $6.1^{*}$ & 6391 & 1904 & 0.30 & 499.0 & 0.000191 & 0.23 & 11.004 & 0.111 & 0.0607 & 0.0002 & 0.0907 & 0.0009 & 559.5 & 5.5 \\
\hline 6.2 & 919 & 784 & 0.85 & 68.3 & 0.000427 & 0.82 & 11.552 & 0.121 & 0.0647 & 0.0004 & 0.0859 & 0.0009 & 531.0 & 5.5 \\
\hline $7.1^{*}$ & 4768 & 495 & 0.10 & 313.8 & 0.003455 & 6.74 & 13.053 & 0.133 & 0.1104 & 0.0008 & 0.0714 & 0.0007 & 444.9 & 4.5 \\
\hline $7.2^{*}$ & 213 & 142 & 0.67 & 26.3 & $\mathrm{e}$ & 0.30 & 6.971 & 0.105 & 0.0703 & 0.0006 & 0.1430 & 0.0022 & 861.7 & 12.5 \\
\hline 8.1 & 458 & 244 & 0.53 & 34.1 & 0.000063 & 0.04 & 11.549 & 0.126 & 0.0584 & 0.0006 & 0.0866 & 0.0010 & 535.1 & 5.7 \\
\hline 9.1 & 275 & 26 & 0.10 & 20.4 & 0.000144 & 0.26 & 11.583 & 0.134 & 0.0602 & 0.0008 & 0.0861 & 0.0010 & 532.5 & 6.0 \\
\hline 10.1 & 481 & 13 & 0.03 & 34.9 & 0.000077 & 0.20 & 11.835 & 0.130 & 0.0594 & 0.0006 & 0.0843 & 0.0009 & 521.9 & 5.6 \\
\hline 11.1 & 725 & 375 & 0.52 & 52.5 & 0.000002 & 0.05 & 11.882 & 0.125 & 0.0582 & 0.0004 & 0.0841 & 0.0009 & 520.6 & 5.4 \\
\hline 12.1 & 771 & 458 & 0.59 & 56.3 & 0.000025 & 0.01 & 11.766 & 0.124 & 0.0579 & 0.0004 & 0.0850 & 0.0009 & 525.8 & 5.4 \\
\hline 13.1 & 337 & 160 & 0.48 & 25.4 & 0.000616 & 1.37 & 11.365 & 0.127 & 0.0694 & 0.0007 & 0.0868 & 0.0010 & 536.4 & 5.9 \\
\hline 14.1 & 296 & 195 & 0.66 & 22.4 & 0.000062 & 0.09 & 11.352 & 0.127 & 0.0591 & 0.0006 & 0.0880 & 0.0010 & 543.8 & 5.9 \\
\hline 15.1 & 600 & 245 & 0.41 & 44.0 & $\mathrm{e}$ & 0.07 & 11.721 & 0.124 & 0.0585 & 0.0004 & 0.0853 & 0.0009 & 527.4 & 5.5 \\
\hline $16.1^{*}$ & 157 & 97 & 0.62 & 12.3 & 0.000321 & 0.38 & 10.979 & 0.131 & 0.0619 & 0.0009 & 0.0907 & 0.0011 & 559.9 & 6.5 \\
\hline $17.1^{*}$ & 2572 & 684 & 0.27 & 175.3 & 0.000473 & 1.03 & 12.605 & 0.128 & 0.0652 & 0.0003 & 0.0785 & 0.0008 & 487.3 & 4.9 \\
\hline 18.1 & 688 & 72 & 0.10 & 50.5 & 0.000161 & 0.30 & 11.719 & 0.123 & 0.0604 & 0.0004 & 0.0851 & 0.0009 & 526.3 & 5.4 \\
\hline $19.1^{*}$ & 1534 & 221 & 0.14 & 108.9 & 0.000326 & 0.63 & 12.094 & 0.125 & 0.0626 & 0.0003 & 0.0822 & 0.0009 & 509.0 & 5.1 \\
\hline 20.1 & 374 & 75 & 0.20 & 27.4 & 0.000091 & 0.01 & 11.702 & 0.128 & 0.0581 & 0.0006 & 0.0854 & 0.0010 & 528.5 & 5.7 \\
\hline Sample NF-I & s-62 Villa Al & pertina gran & & & & & & & & & & & & \\
\hline 1.1 & 3349 & 236 & 0.07 & 246.6 & 0.000013 & $<0.01$ & 11.665 & 0.118 & 0.0579 & 0.0002 & 0.0857 & 0.0009 & 530.3 & 5.3 \\
\hline 2.1 & 242 & 135 & 0.56 & 17.8 & $\mathrm{e}$ & 0.22 & 11.655 & 0.132 & 0.0598 & 0.0007 & 0.0856 & 0.0010 & 529.5 & 5.9 \\
\hline $3.1^{*}$ & 452 & 549 & 1.22 & 39.9 & 0.000038 & 0.15 & 9.729 & 0.104 & 0.0620 & 0.0005 & 0.1026 & 0.0011 & 629.8 & 6.6 \\
\hline $4.1^{*}$ & 304 & 141 & 0.46 & 21.3 & 0.000208 & 0.19 & 12.247 & 0.136 & 0.0589 & 0.0006 & 0.0815 & 0.0009 & 505.1 & 5.5 \\
\hline 1.2 & 283 & 149 & 0.53 & 20.8 & 0.000032 & 0.11 & 11.733 & 0.132 & 0.0588 & 0.0007 & 0.0851 & 0.0010 & 526.7 & 5.8 \\
\hline $5.1^{*}$ & 1689 & 340 & 0.20 & 128.8 & 0.004133 & 7.48 & 11.261 & 0.115 & 0.1183 & 0.0012 & 0.0822 & 0.0009 & 509.0 & 5.2 \\
\hline 6.1 & 633 & 319 & 0.50 & 46.8 & 0.000337 & 0.61 & 11.615 & 0.124 & 0.0629 & 0.0004 & 0.0856 & 0.0009 & 529.3 & 5.5 \\
\hline
\end{tabular}

Uncertainties given at the $1 \mathrm{~s}$ level.

Error in Temora reference zircon calibration was $0.31 \%$ for the analytical session (not included in above errors but required when comparing data from different mounts) f206\% denotes the percentage of $206 \mathrm{~Pb}$ that is common $\mathrm{Pb}$.

Correction for common $\mathrm{Pb}$ made using the measured $238 \mathrm{U} / 206 \mathrm{~Pb}$ and $207 \mathrm{~Pb} / 206 \mathrm{~Pb}$ ratios as outlined in Williams (1998).

* indicates analysis not included in age calculation.

González et al. (1985), Massabie et al. (2002) and Koukharsky et al. (2003). Many of the earlier ages are KeAr (whole-rock), which are show a wide range and are not reliable for dating the crystalliza-tion of the granitic rocks. A RbeSr isochron of $494 \pm 11 \mathrm{Ma}$ for the Granitic Porphyry of Oncán (north of Sierra Norte) presented by Rapela et al. (1991), is one of the youngest ages for crystallization within the batholith. UePb zircon ages are more robust and more useful for delimiting the period of development of the magmatic arc, which is the approach adopted here. Published UePb data are considered in Section 9.1 after presentation of the new analytical results.
One sample of the pre-/syn-mylonitic Juan García granodiorite (NF-Is-23), which is considered contemporaneous with the Ischilín porphyritic granite, and two samples of the post-mylonitic Villa Albertina granite (NF-Is-20 and -62) were dated by the $\mathrm{UePb}$ SHRIMP zircon method. The results are plotted in Fig. 7. In addition, detrital zircons were analysed from a gneiss with granitic veins, representing the metamorphic basement host rock to investigate its provenance; this sample comes from El Pueblito $\left(29^{\circ} 10^{\circ} 9.6^{00} \mathrm{~S}\right.$, $63^{\circ} 34^{0} 57.3^{00} \mathrm{~W}$ ), about $150 \mathrm{~km}$ north of the igneous rocks analysed here, where the host rocks to the batholith are well exposed in quarries. 


\begin{tabular}{|c|c|c|c|c|c|c|c|c|c|c|c|c|c|c|c|c|c|c|c|c|c|c|}
\hline \multirow{3}{*}{$\begin{array}{l}\begin{array}{l}\text { Grain } \\
\text { spot }\end{array} \\
1.1\end{array}$} & \multirow{3}{*}{$\begin{array}{l}\mathrm{U}(\mathrm{ppm}) \\
434\end{array}$} & \multirow{3}{*}{$\begin{array}{l}\text { Th (ppm) } \\
120\end{array}$} & \multirow{2}{*}{\multicolumn{2}{|c|}{$\mathrm{Th} / \mathrm{U}^{206} \mathrm{~Pb}^{*}(\mathrm{ppm})$}} & \multirow{3}{*}{$\frac{{ }^{204} \mathrm{~Pb} /{ }^{206} \mathrm{~Pb}}{0.000581}$} & \multirow{3}{*}{$\begin{array}{r}\text { f206\% } \\
0.64\end{array}$} & \multicolumn{6}{|c|}{ Measured ratios } & \multicolumn{4}{|c|}{ Radiogenic ratios } & \multirow[t]{3}{*}{$\mathrm{r}$} & Ages & 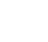 & 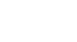 & 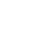 & \\
\hline & & & & & & & ${ }^{238} \mathrm{U}^{206}$ & & ${ }^{207} \mathrm{~Pb} /{ }^{2 \mathrm{C}}$ & & ${ }^{206} \mathrm{~Pb} /{ }^{23}$ & & ${ }^{207} \mathrm{~Pb} /{ }^{23}$ & & ${ }^{207} \mathrm{~Pb} /{ }^{20}$ & & & ${ }^{206} \mathrm{~Pb}$ & $\pm^{20}$ & ${ }^{3} \mathrm{~Pb} /{ }^{206}$ & \%Dis & \\
\hline & & & 0.28 & 36 & & & 10.302 & 0.124 & 0.0650 & 0.0007 & 0.0964 & 0.0012 & & & & & & 594 & 7 & & & \\
\hline 2.1 & 72 & 98 & 1.36 & 12 & 0.000519 & 0.87 & 5.196 & 0.093 & 0.0811 & 0.0015 & 0.1908 & 0.0035 & 1.938 & 0.092 & 0.0737 & 0.0032 & 0.386 & 1126 & 19 & 1033 & 88 & \\
\hline 3.1 & 131 & 58 & 0.44 & 10 & 0.001517 & 1.49 & 11.349 & 0.188 & 0.0703 & 0.0015 & 0.0868 & 0.0015 & & & & & & 537 & 9 & & & \\
\hline 4.1 & 351 & 79 & 0.23 & 30 & 0.000202 & 0.09 & 10.013 & 0.128 & 0.0610 & 0.0008 & 0.0998 & 0.0013 & & & & & & 613 & 8 & & & \\
\hline 5.1 & 260 & 203 & 0.78 & 23 & 0.000050 & 0.28 & 9.895 & 0.134 & 0.0627 & 0.0010 & 0.1008 & 0.0014 & & & & & & 619 & 8 & & & \\
\hline 6.1 & 97 & 102 & 1.06 & 8 & $\mathrm{e}$ & 0.10 & 10.479 & 0.185 & 0.0604 & 0.0015 & 0.0953 & 0.0017 & & & & & & 587 & 10 & & & \\
\hline 7.1 & 583 & 404 & 0.69 & 56 & 0.000061 & 0.21 & 8.890 & 0.103 & 0.0640 & 0.0006 & 0.1123 & 0.0013 & & & & & & 686 & 8 & & & \\
\hline 8.1 & 334 & 579 & 1.73 & 26 & 0.000710 & 0.83 & 11.079 & 0.143 & 0.0654 & 0.0011 & 0.0895 & 0.0012 & & & & & & 553 & 7 & & & \\
\hline 9.1 & 105 & 64 & 0.61 & 15 & 0.000210 & 0.36 & 6.132 & 0.098 & 0.0749 & 0.0012 & 0.1625 & 0.0026 & 1.612 & 0.051 & 0.0719 & 0.0020 & 0.509 & 971 & 15 & 984 & 55 & 1 \\
\hline 10.1 & 143 & 43 & 0.30 & 53 & 0.000041 & 0.06 & 2.311 & 0.032 & 0.2150 & 0.0018 & 0.4325 & 0.0060 & 12.79 & 0.208 & 0.2145 & 0.0018 & 0.852 & 2317 & 27 & 2940 & 14 & 21 \\
\hline 11.1 & 318 & 233 & 0.73 & 86 & 0.000078 & 0.12 & 3.177 & 0.038 & 0.1143 & 0.0007 & 0.3143 & 0.0038 & 4.908 & 0.069 & 0.1132 & 0.0008 & 0.852 & 1762 & 19 & 1852 & 13 & 5 \\
\hline 12.1 & 231 & 152 & 0.66 & 18 & 0.000131 & 0.34 & 10.877 & 0.150 & 0.0617 & 0.0010 & 0.0916 & 0.0013 & & & & & & 565 & 8 & & & \\
\hline 13.1 & 563 & 220 & 0.39 & 79 & 0.000096 & 0.17 & 6.141 & 0.070 & 0.0756 & 0.0006 & 0.1626 & 0.0019 & 1.663 & 0.025 & 0.0742 & 0.0007 & 0.750 & 971 & 10 & 1047 & 20 & 7 \\
\hline 14.1 & 521 & 387 & 0.74 & 43 & 0.000018 & 0.04 & 10.491 & 0.124 & 0.0599 & 0.0007 & 0.0953 & 0.0011 & & & & & & 587 & 7 & & & \\
\hline 15.1 & 151 & 205 & 1.36 & 13 & 0.000747 & 0.27 & 9.947 & 0.162 & 0.0626 & 0.0014 & 0.1003 & 0.0017 & & & & & & 616 & 10 & & & \\
\hline 16.1 & 457 & 286 & 0.63 & 36 & 0.000324 & 0.24 & 10.947 & 0.133 & 0.0608 & 0.0007 & 0.0911 & 0.0011 & & & & & & 562 & 7 & & & \\
\hline 17.1 & 164 & 85 & 0.52 & 36 & 0.000042 & 0.07 & 3.894 & 0.054 & 0.0956 & 0.0009 & 0.2566 & 0.0035 & 3.363 & 0.059 & 0.0951 & 0.0010 & 0.782 & 1472 & 18 & 1529 & 21 & 4 \\
\hline 18.1 & 86 & 88 & 1.03 & 7 & $\mathrm{e}$ & 0.32 & 10.834 & 0.198 & 0.0616 & 0.0016 & 0.0920 & 0.0017 & & & & & & 567 & 10 & & & \\
\hline 19.1 & 273 & 163 & 0.60 & 107 & $\mathrm{e}$ & $<0.01$ & 2.187 & 0.027 & 0.1820 & 0.0008 & 0.4574 & 0.0056 & 11.49 & 0.150 & 0.1822 & 0.0009 & 0.932 & 2428 & 25 & 2673 & 8 & 9 \\
\hline 20.1 & 122 & 126 & 1.02 & 10 & $\mathrm{e}$ & 0.24 & 11.076 & 0.181 & 0.0607 & 0.0013 & 0.0901 & 0.0015 & & & & & & 556 & 9 & & & \\
\hline 21.1 & 154 & 231 & 1.50 & 14 & 0.000338 & $<0.01$ & 9.733 & 0.146 & 0.0603 & 0.0011 & 0.1028 & 0.0016 & & & & & & 631 & 9 & & & \\
\hline 22.1 & 640 & 563 & 0.88 & 53 & 0.000086 & 0.20 & 10.384 & 0.119 & 0.0613 & 0.0006 & 0.0961 & 0.0011 & & & & & & 592 & 7 & & & \\
\hline 23.1 & 260 & 37 & 0.14 & 36 & 0.000013 & 0.02 & 6.222 & 0.080 & 0.0720 & 0.0008 & 0.1607 & 0.0021 & 1.591 & 0.028 & 0.0718 & 0.0008 & 0.744 & 961 & 11 & 981 & 24 & 2 \\
\hline 24.1 & 307 & 155 & 0.51 & 81 & 0.000068 & 0.11 & 3.265 & 0.039 & 0.1165 & 0.0007 & 0.3059 & 0.0037 & 4.873 & 0.067 & 0.1155 & 0.0008 & 0.876 & 1721 & 18 & 1888 & 12 & 9 \\
\hline 25.1 & 111 & 178 & 1.60 & 9 & 0.000797 & 0.18 & 11.063 & 0.188 & 0.0602 & 0.0014 & 0.0902 & 0.0016 & & & & & & 557 & 9 & & & \\
\hline 26.1 & 622 & 27 & 0.04 & 57 & 0.000000 & 0.04 & 9.459 & 0.109 & 0.0615 & 0.0006 & 0.1057 & 0.0012 & & & & & & 648 & 7 & & & \\
\hline 27.1 & 409 & 539 & 1.32 & 53 & 0.000102 & 0.18 & 6.594 & 0.078 & 0.0706 & 0.0006 & 0.1514 & 0.0018 & 1.443 & 0.025 & 0.0691 & 0.0009 & 0.678 & 909 & 10 & 902 & 27 & \\
\hline 28.1 & 298 & 329 & 1.11 & 24 & 0.000091 & 0.23 & 10.795 & 0.140 & 0.0609 & 0.0009 & 0.0924 & 0.0012 & & & & & & 570 & 7 & & & \\
\hline 29.1 & 301 & 156 & 0.52 & 83 & 0.000135 & 0.21 & 3.134 & 0.038 & 0.1212 & 0.0007 & 0.3184 & 0.0039 & 5.240 & 0.074 & 0.1194 & 0.0009 & 0.860 & 1782 & 19 & 1947 & 13 & 8 \\
\hline 30.1 & 214 & 103 & 0.48 & 18 & 0.000367 & 0.09 & 10.031 & 0.145 & 0.0610 & 0.0011 & 0.0996 & 0.0015 & & & & & & 612 & 9 & & & \\
\hline 31.1 & 261 & 208 & 0.80 & 22 & 0.000023 & $<0.01$ & 10.351 & 0.138 & 0.0595 & 0.0009 & 0.0966 & 0.0013 & & & & & & 595 & 8 & & & \\
\hline 32.1 & 364 & 164 & 0.45 & 94 & 0.000234 & 0.37 & 3.324 & 0.040 & 0.1154 & 0.0007 & 0.2997 & 0.0036 & 4.639 & 0.070 & 0.1123 & 0.0010 & 0.800 & 1690 & 18 & 1836 & 16 & 8 \\
\hline 33.1 & 159 & 81 & 0.51 & 11 & 0.004728 & 7.73 & 12.380 & 0.190 & 0.1190 & 0.0037 & 0.0745 & 0.0012 & & & & & & 463 & 7 & & & \\
\hline 34.1 & 641 & 43 & 0.07 & 176 & 0.000076 & 0.12 & 3.122 & 0.035 & 0.1203 & 0.0005 & 0.3199 & 0.0035 & 5.263 & 0.064 & 0.1193 & 0.0006 & 0.918 & 1789 & 17 & 1946 & 9 & 8 \\
\hline 35.1 & 138 & 23 & 0.17 & 19 & 0.000521 & 0.89 & 6.316 & 0.094 & 0.0756 & 0.0011 & 0.1577 & 0.0024 & 1.570 & 0.046 & 0.0722 & 0.0015 & 0.717 & 944 & 13 & 992 & 43 & 5 \\
\hline 36.1 & 177 & 126 & 0.71 & 22 & 0.000594 & 1.03 & 7.069 & 0.103 & 0.0779 & 0.0012 & 0.1400 & 0.0021 & 1.340 & 0.057 & 0.0694 & 0.0027 & 0.354 & 845 & 12 & 911 & 81 & 7 \\
\hline 37.1 & 634 & 208 & 0.33 & 183 & 0.000125 & 0.19 & 2.968 & 0.033 & 0.1222 & 0.0008 & 0.3363 & 0.0037 & 5.589 & 0.073 & 0.1205 & 0.0008 & 0.847 & 1869 & 18 & 1964 & 12 & 5 \\
\hline 38.1 & 487 & 372 & 0.76 & 43 & 0.000095 & 0.01 & 9.806 & 0.117 & 0.0607 & 0.0007 & 0.1020 & 0.0012 & & & & & & 626 & 7 & & & \\
\hline 39.1 & 582 & 45 & 0.08 & 96 & 0.000023 & 0.04 & 5.233 & 0.060 & 0.0773 & 0.0006 & 0.1910 & 0.0022 & 2.028 & 0.028 & 0.0770 & 0.0006 & 0.833 & 1127 & 12 & 1121 & 15 & \\
\hline 40.1 & 463 & 147 & 0.32 & 62 & 0.000014 & 0.02 & 6.371 & 0.076 & 0.0730 & 0.0007 & 0.1569 & 0.0019 & 1.575 & 0.024 & 0.0728 & 0.0007 & 0.784 & 940 & 10 & 1008 & 19 & 7 \\
\hline 41.1 & 284 & 358 & 1.26 & 39 & 0.000002 & $<0.01$ & 6.216 & 0.079 & 0.0741 & 0.0008 & 0.1609 & 0.0021 & 1.644 & 0.028 & 0.0741 & 0.0008 & 0.762 & 962 & 11 & 1044 & 22 & 8 \\
\hline 42.1 & 356 & 317 & 0.89 & 30 & $\mathrm{e}$ & $<0.01$ & 10.034 & 0.129 & 0.0595 & 0.0008 & 0.0998 & 0.0013 & & & & & & 613 & 8 & & & \\
\hline 43.1 & 189 & 123 & 0.65 & 27 & 0.000185 & 0.32 & 6.013 & 0.084 & 0.0721 & 0.0009 & 0.1658 & 0.0023 & 1.588 & 0.040 & 0.0695 & 0.0014 & 0.561 & 989 & 13 & 912 & 43 & \\
\hline 44.1 & 685 & 131 & 0.19 & 102 & 0.000253 & 0.43 & 5.768 & 0.065 & 0.0751 & 0.0005 & 0.1726 & 0.0020 & 1.701 & 0.029 & 0.0715 & 0.0009 & 0.667 & 1027 & 11 & 971 & 26 & \\
\hline 45.1 & 632 & 54 & 0.08 & 88 & 0.000047 & 0.08 & 6.194 & 0.070 & 0.0735 & 0.0005 & 0.1613 & 0.0018 & 1.620 & 0.025 & 0.0728 & 0.0008 & 0.721 & 964 & 10 & 1009 & 22 & 4 \\
\hline 46.1 & 96 & 79 & 0.82 & 8 & 0.000361 & 0.39 & 10.499 & 0.192 & 0.0627 & 0.0016 & 0.0949 & 0.0018 & & & & & & 584 & 10 & & & \\
\hline 47.1 & 373 & 313 & 0.84 & 32 & 0.000322 & 0.81 & 10.019 & 0.125 & 0.0667 & 0.0008 & 0.0990 & 0.0013 & & & & & & 609 & 7 & & & \\
\hline 48.1 & 279 & 233 & 0.83 & 23 & 0.000120 & 0.11 & 10.191 & 0.138 & 0.0609 & 0.0009 & 0.0980 & 0.0014 & & & & & & 603 & 8 & & & \\
\hline 49.1 & 290 & 113 & 0.39 & 42 & 0.000043 & 0.07 & 5.888 & 0.074 & 0.0730 & 0.0008 & 0.1697 & 0.0021 & 1.695 & 0.029 & 0.0724 & 0.0008 & 0.749 & 1011 & 12 & 998 & & \\
\hline 51.1 & 491 & 404 & 0.82 & 132 & 0.000066 & 0.10 & 3.202 & 0.037 & 0.1294 & 0.0008 & 0.3120 & 0.0036 & 5.527 & 0.073 & 0.1285 & 0.0009 & 0.863 & 1750 & 17 & 2077 & & 16 \\
\hline 52.1 & 590 & 101 & 0.17 & 69 & 0.000632 & 1.10 & 7.321 & 0.087 & 0.0730 & 0.0007 & 0.1351 & 0.0016 & 1.189 & 0.034 & 0.0639 & 0.0016 & 0.422 & 817 & 9 & 737 & & -11 \\
\hline 53.1 & 762 & 371 & 0.49 & 60 & 0.001330 & 1.99 & 10.982 & 0.124 & 0.0748 & 0.0020 & 0.0892 & 0.0011 & & & & & & 551 & 6 & & & \\
\hline 54.1 & 435 & 473 & 1.09 & 36 & 0.000539 & 0.70 & 10.415 & 0.128 & 0.0653 & 0.0008 & 0.0953 & 0.0012 & & & & & & 587 & 7 & & & \\
\hline 55.1 & 175 & 73 & 0.41 & 40 & 0.000141 & 0.23 & 3.734 & 0.053 & 0.1052 & 0.0010 & 0.2672 & 0.0038 & 3.805 & 0.088 & 0.1033 & 0.0019 & 0.614 & 1527 & 19 & 1684 & 34 & 9 \\
\hline 56.1 & 328 & 101 & 0.31 & 30 & 0.001111 & 1.57 & 9.427 & 0.126 & 0.0739 & 0.0010 & 0.1044 & 0.0014 & & & & & & 640 & 8 & & & \\
\hline
\end{tabular}




\subsection{Age of the granitic rocks}

Individual grain ages obtained for the Juan García granodiorite range $488 \mathrm{e} 605$ $\mathrm{Ma}$ (Table 3, Fig. 7). However the three youngest ages require large common- $\mathrm{Pb}$ corrections and the two oldest appear to be anomalous, probably due to inheritance effects. The remaining 16 ages are tightly grouped and give a weighted mean of $537 \pm 4 \mathrm{Ma}$ (MSWD 1/4 1.2, uncertainty including that of the calibration as in Table 3). This is interpreted as the age of igneous crystallization, placing this pre- to syn-deformational magmatic event in the earliest Cambrian, equivalent to the Fortunian stage (International Stratigraphic Chart, IUGS, 2009).

Sample NF-Is-20 from the Villa Albertina granite yielded UePb SHRIMP zircon ages with a wide range of $445 \mathrm{e} 862 \mathrm{Ma}$ (Table 3 ). Again, several of the outlying values may be ignored on the grounds of high common- $\mathrm{Pb}$ corrections, whereas those older than about 550 Ma may be ascribed to inheritance (Fig. 7). Twelve of the ages lie between 520 and $545 \mathrm{Ma}$ and give a weighted mean of $530 \pm 5 \mathrm{Ma}$ (MSWD $1 / 41.6$ ). Only seven grains were analysed from the second sample (NFIs-62), of which one is clearly inherited and four cluster very tightly at $529 \mathrm{e} 530 \mathrm{Ma}$ (Table 3). In view of the obvious concordance of predominant ages in the two samples, it was considered acceptable to combine the datasets (Fig. 7), yielding a weighted mean for 16 grains of $530 \pm 4$ Ma (MSWD 1/4 1.2) as a best estimate of the crystallization age. Although slightly younger than the age of the Juan Garcia granodiorite, this is scarcely distinguishable within analytical uncertainty and also corresponds to very early Cambrian, certainly within the Terreneuvian epoch (International Stratigraphic Chart, IUGS, 2009).

\subsection{Provenance of the metasedimentary country rocks}

The ages of detrital zircons in metasedimentary rock sample SNO-10034 were determined (Table 4). Plotting in the TeraeWasserburg diagram (Fig. 8a) shows a wide spread of data. For ages $>800 \mathrm{Ma}$, common Pb correction was made using the measured ${ }^{204} \mathrm{~Pb}$ so that concordance could be assessed e for younger ages the correction was made using ${ }^{207} \mathrm{~Pb}$ so that this test was not possible, although the youngest apparent age (for grain 33 ) is discounted on the grounds of a high common $\mathrm{Pb}$ content. A probability diagram for the more robust ages (Fig. 8b) shows strong peaks at $560 \mathrm{Ma}, 600 \mathrm{Ma}$ and ca. $970 \mathrm{Ma}$, with subsidiary minor concentrations at ca. $1850 \mathrm{Ma}$ and $1950 \mathrm{Ma}$ and scattered intermediate ages including one at ca. $2670 \mathrm{Ma}$. The youngest peak of $560 \mathrm{Ma}$ gives a maximum age for sedimentary deposition of the protolith.

8.

$\mathrm{Sr}$ and $\mathrm{Nd}$ isotope analyses

The initial ${ }^{87} \mathrm{Sr} /{ }^{86} \mathrm{Sr}$ ratios (at $537 \mathrm{Ma}$ ) of the pre-/syn-mylonitic

units are somewhat variable, ranging from 0.7060 to 0.7113 , and the initial $\varepsilon \mathrm{Nd}$ values vary from -5.5 to -1.8 (Table 5 , Fig. 9). The least evolved samples are the La Maroma granodiorite with initial ${ }^{87} \mathrm{Sr} /{ }^{86} \mathrm{Sr}$ of 0.7060 and $\varepsilon N d t$ of $-1,8$ and the micro-tonalite dyke $\left(0.7062\right.$ and -2.5 , respectively). The initial ${ }^{87} \mathrm{Sr} /{ }^{86} \mathrm{Sr}$ for the Ischilín and Juan García granites are more radiogenic (0.7090e0.7109), ignoring one sample for which the very high $\mathrm{Rb} / \mathrm{Sr}$ ratios requires too great an age correction (which is therefore very uncertain), but rather more variable $\varepsilon N d t$ values $(-2.0$ to -5.5$)$. The initial ${ }^{87} \mathrm{Sr} /{ }^{86} \mathrm{Sr}$ ratios (at $530 \mathrm{Ma}$ ) of the postmylonitic Villa Albertina granite are rather invariant at $0.7083 \mathrm{e} 0.7100$ and the $\varepsilon N d t$ values even more so at -5.7 to -5.9 , and are comparable to the metamorphic base-ment sample at 0.7113 and -5.0 , respectively. Thus the negative $\varepsilon N d t$ and relatively high initial ${ }^{87} \mathrm{Sr} /{ }^{86} \mathrm{Sr}$ ratios show that both pre-/synand post-mylonitic units all have strong crustal signature and seem to define a trend towards the basement composition. SmeNd 
Table 5

Isotopic analyses of samples from the southern sector of Sierra Norte-Ambargasta batholith.

\begin{tabular}{|c|c|c|c|c|c|c|c|c|c|}
\hline Unit & \multirow{2}{*}{$\frac{\text { Sample }}{\text { NF Is } 8}$} & \multicolumn{2}{|l|}{$\mathrm{Rb}(\mathrm{ppm})$} & \multicolumn{2}{|l|}{$\mathrm{Sr}(\mathrm{ppm})$} & \multicolumn{2}{|l|}{${ }^{87} \mathrm{Rb} /{ }^{86} \mathrm{Sr}$} & ${ }^{87} \mathrm{Sr} /{ }^{86} \mathrm{Sr}$ & $\left({ }^{87} \mathrm{Sr} /{ }^{86} \mathrm{Sr}\right)_{\mathrm{t}}$ \\
\hline Villa Albertina granite & & 115.3 & \multicolumn{3}{|c|}{274.9} & \multicolumn{2}{|l|}{1.215} & 0.71870 & 0.7095 \\
\hline Villa Albertina granite & NF Is 13 & \multicolumn{2}{|l|}{118.6} & \multicolumn{2}{|l|}{210.2} & \multicolumn{2}{|l|}{1.635} & 0.72240 & 0.7101 \\
\hline Villa Albertina granite & NF Is 20 & \multicolumn{2}{|l|}{123.6} & \multicolumn{2}{|l|}{217.6} & \multicolumn{2}{|l|}{1.646} & 0.72230 & 0.7099 \\
\hline Villa Albertina granite & NF Is 62 & 145.3 & & \multicolumn{2}{|l|}{291.2} & \multicolumn{2}{|l|}{1.445} & 0.71980 & 0.7089 \\
\hline Villa Albertina granite & NF Is 108 & 197.9 & & \multicolumn{2}{|l|}{99.5} & \multicolumn{2}{|l|}{5.779} & 0.75200 & 0.7083 \\
\hline Juan García granite Ischilín & NF Is 23 & 169.6 & & \multicolumn{2}{|l|}{163} & \multicolumn{2}{|l|}{3.018} & 0.73370 & 0.7109 \\
\hline porphyritic granite Ischilín & NF Is 117 & 140.1 & & \multicolumn{2}{|l|}{254} & \multicolumn{2}{|l|}{1.600} & 0.73590 & 0.7237 \\
\hline porphyritic granite La & NF Is 119 & 131.9 & & 156.1 & & 2.450 & & 0.72860 & 0.7098 \\
\hline Maroma granite Aplite dyke & NF Is 134 & 134.4 & & 154.3 & & 2.524 & & 0.72530 & 0.7060 \\
\hline Micro-tonalite dyke & NF Is 86 & 76.5 & & 101.9 & & 2.175 & & 0.72370 & 0.7070 \\
\hline Metamorphic basement & NF Is 132 & 62.8 & & 398.3 & & 0.456 & & 0.70970 & 0.7062 \\
\hline & NF Is 138 & 158.3 & & 148.1 & & 3.101 & & 0.73500 & 0.7113 \\
\hline Unit & Sample & Sm (ppm) & $\mathrm{Nd}(\mathrm{ppm})$ & & ${ }^{147} \mathrm{Sm} /{ }^{144} \mathrm{Nd}$ & & ${ }^{143} \mathrm{Nd} /{ }^{144} \mathrm{Nd}$ & & $\mathrm{T}_{\mathrm{DM}}(\mathrm{Ma})$ \\
\hline Villa Albertina granite & NF Is 8 & 4.55 & 24.19 & & 0.1137 & & 0.51206 & -5.7 & 1677 \\
\hline Villa Albertina granite & NF Is 13 & 4.48 & 23.86 & & 0.1134 & & 0.51205 & -5.8 & 1690 \\
\hline Villa Albertina granite & NF Is 20 & 4.05 & 21.38 & & 0.1144 & & 0.51205 & -5.9 & 1695 \\
\hline Juan García granite Ischilín & NF Is 23 & 5.16 & 24.64 & & 0.1266 & & 0.51211 & -5.5 & 1670 \\
\hline porphyritic granite Ischilín & NF Is 117 & 11.53 & 52.90 & & 0.1318 & & 0.51231 & -1.9 & 1403 \\
\hline porphyritic granite La & NF Is 119 & 4.94 & 24.53 & & 0.1216 & & 0.51210 & -5.4 & 1660 \\
\hline Maroma granite Aplite dyke & NF Is 134 & 7.14 & 30.66 & & 0.1408 & & 0.51235 & -1.8 & 1390 \\
\hline Micro-tonalite dyke & NF Is 86 & 5.17 & 19.93 & & 0.1569 & & 0.51228 & -4.3 & 1579 \\
\hline Metamorphic basement & NF Is 132 & 2.49 & 12.84 & & 0.1171 & & 0.51223 & -2.5 & 1446 \\
\hline Metamorphic basement & NF Is 138 & 8.81 & 49.98 & & 0.1065 & & 0.51206 & -5.1 & 1641 \\
\hline & NF Is 115 & 6.03 & 30.91 & & 0.1179 & & 0.51203 & $\begin{array}{l}-0.1 \\
-6.5 \\
\end{array}$ & 1739 \\
\hline
\end{tabular}

Estimated 1-S analytical uncertainties: $0.01 \%$ for ${ }^{87} \mathrm{Sr} /{ }^{86} \mathrm{Sr}, 1 \%$ for ${ }^{87} \mathrm{Rb} /{ }^{86} \mathrm{Sr}, 0.006 \%$ for ${ }^{143} \mathrm{Nd} /{ }^{144} \mathrm{Nd}$ and $0.1 \%$ for ${ }^{147} \mathrm{Sm} /{ }^{144} \mathrm{Nd}$.

Twelve analyses of NBS987 gave a mean ${ }^{87} \mathrm{Sr} /{ }^{86} \mathrm{Sr}$ of $0.710176 \pm 0.00009$.

Six analyses of the La Jolla Nd standard gave a mean ${ }^{143} \mathrm{Nd} /{ }^{144} \mathrm{Nd}$ of $0.511838 \pm 0.00003$

Initial isotopic compositions calculated assuming an age of $537 \mathrm{Ma}$ for the pre-/syn-mylonitic rocks and 530 Ma for the Villa Albertina granite. $\mathrm{T}_{\mathrm{DM}}$ model ages according to DePaolo et al. (1991).

model ages ( $\mathrm{T}_{\mathrm{DM}}{ }^{*}$, following the model of DePaolo et al. (1991)) vary between $1.38 \mathrm{Ga}$ (Mesoproterozoic) for the La Maroma granodiorite and $1.70 \mathrm{Ga}$ (Paleoproterozoic) for the Villa Albertina granite.

\section{Discussion}

9.1. The chronology of magmatism in the Sierra NorteAmbargasta batholith

Table 6 summarizes published conventional $\mathrm{UePb}$ and $\mathrm{UePb}$ SHRIMP ages of granitic units in different localities of the Sierra Norte-Ambargasta batholith. Some of these ages are Precambrian (>550 Ma), whereas others are as young as $515 \pm 4 \mathrm{M}$ a ( StuartSmith et al., 1999). However, there is a reasonable concentration

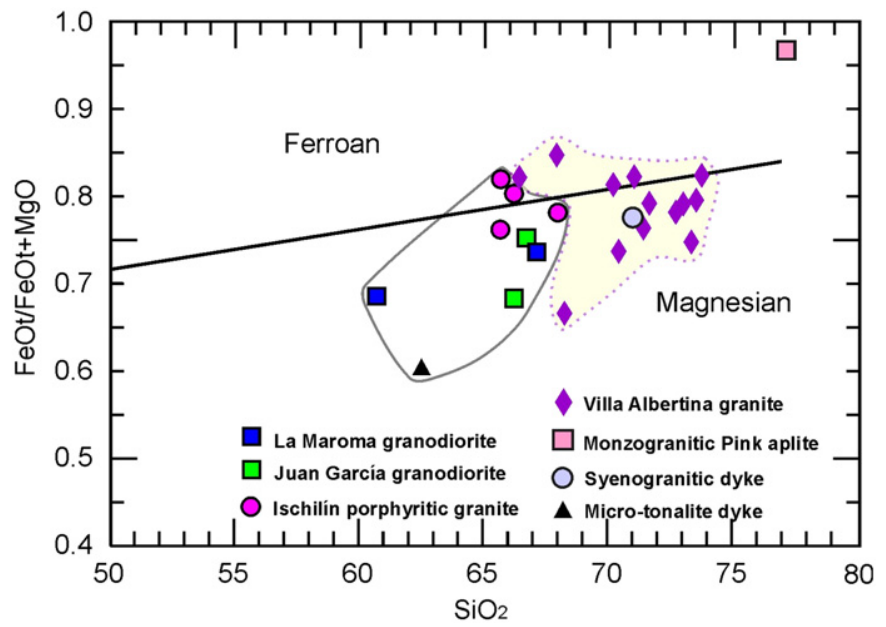

Fig. 4. Diagram of Frost et al. (2001) for samples of the southern Sierra NorteAmbargasta batholith. The plutonic rocks are predominantly magnesian granitoids. of $\mathrm{UePb}$ zircon ages in the range $530 \mathrm{e} 540 \mathrm{Ma}$, now reinforced by the data presented in this paper. The results for the Los Burros dacite include an age of (effectively) $532 \pm 2 \mathrm{Ma}$, which Leal et al.(2003) interpreted as due to inheritance, preferring one of the younger age grouping at $512 \mathrm{Ma}$ as referring to crystallization: in the light of the new results we would question that interpretation. Some of the older ages are based on discordant data in which the ${ }^{207} \mathrm{~Pb} /{ }^{206} \mathrm{~Pb}$ age is favoured by forcing a discordia through zero in the Concordia diagram e the ${ }^{206} \mathrm{~Pb} /{ }^{238} \mathrm{U}$ ages are often much closer to $530 \mathrm{e} 540$ Ma, e.g., the data presented by Söllner et al. (2000). Thus, although there is evidence for late Neoproterozoic magma-tism, perhaps contemporaneous with deposition of the sedimen-tary protoliths of the metamorphic basement, there is now

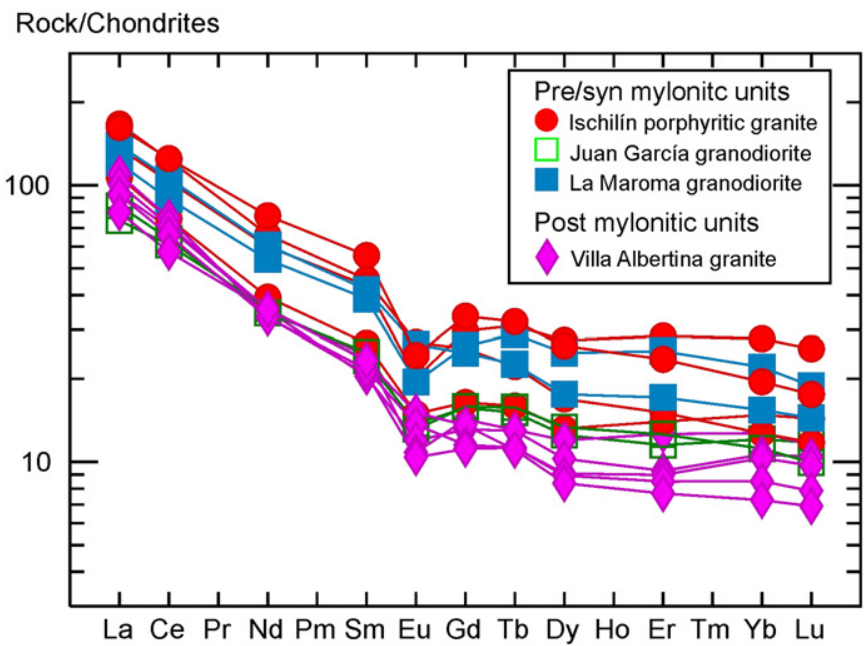

Fig. 5. Rare-earth element diagrams normalized to chondrites according to Nakamura (1974): Ischilín porphyritic granite; Juan García granodiorite; La Maroma granodiorite; Villa Albertina granite. 


\section{Rock/Chondrites}

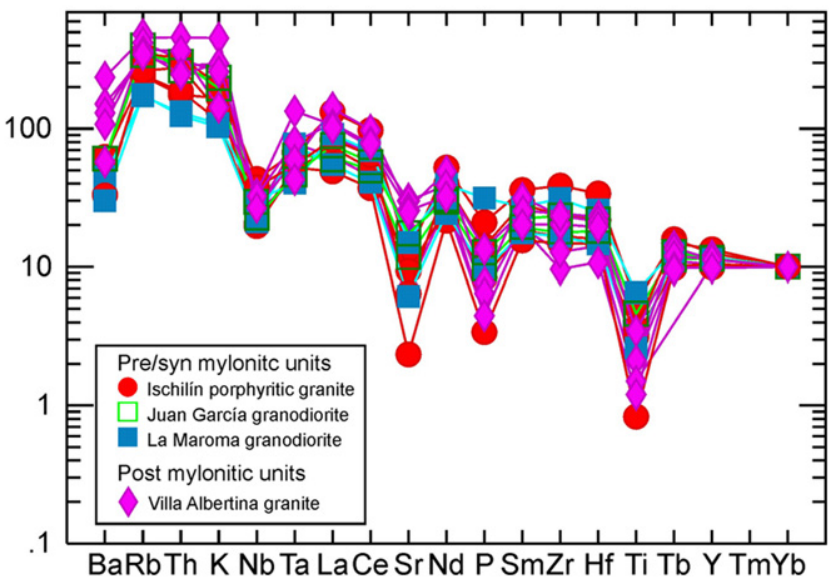

Fig. 6. Multi-element diagram after Thompson (1982). The plutons of the southern Sierra Norte-Ambargasta batholith show a volcanic arc geochemical signature.
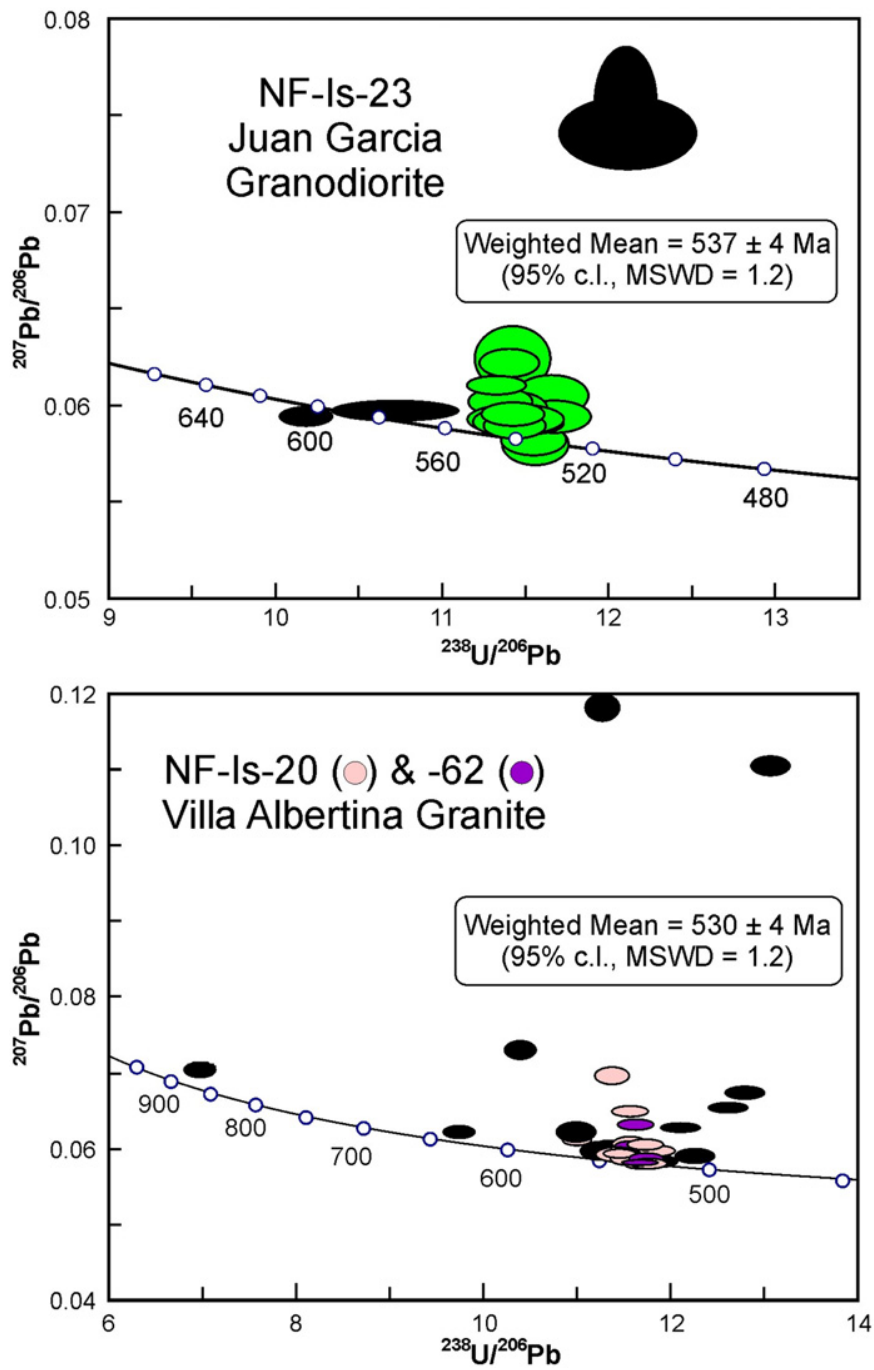

Fig. 7. TeraeWasserburg UePb Concordia diagrams for SHRIMP zircon data for (a) the pre-/syn-mylonitic Juan García granodiorite-one analysis plots above the field shown;(b) two samples of the Villa Albertina post-mylonitic granite. All error ellipses are at $68.3 \%$ confidence limits, grey ellipses indicate data not included in the age calculations since they are thought to show either inheritance or Pb-loss. Uncertainties on final ages include those in the calibration. a convincing body of data that demonstrate emplacement of the major plutonic rocks of the batholith in Early Cambrian times. This is especially true of the granitoids associated with the development of the mylonite belts presented here.

\subsection{Relationship of granites to the Sauce Puncoelschilín mylonitic belt}

The igneous and metamorphic units that compose the southern sector of the Sierra Norte-Ambargasta batholith have been described based on their time and space relationship with the Ischilín mylonitic belt, which Martino (2003) considered to be related to the a similar belt farther north in Sierra de Sauce Punco (Figs. 1 and 2). Martino (2003) also proposed a preOrdovician age for deformation, as the mylonitic belt does not cut across the $494 \pm \mathbf{4}$ Ma Granitic Porphyry of Oncán of Rapela et al. (1991). Our detailed study in the Ischilin area confirms the correlation of these shear zones, for which we propose the name Sauce Puncoelschilín mylonitic belt.

On the other hand, a recent structural study of Sierra Norte de Córdoba recognized a two-stage compressive deformational history during the Early Cambrian (Von Gosen and Prozzi, 2010).
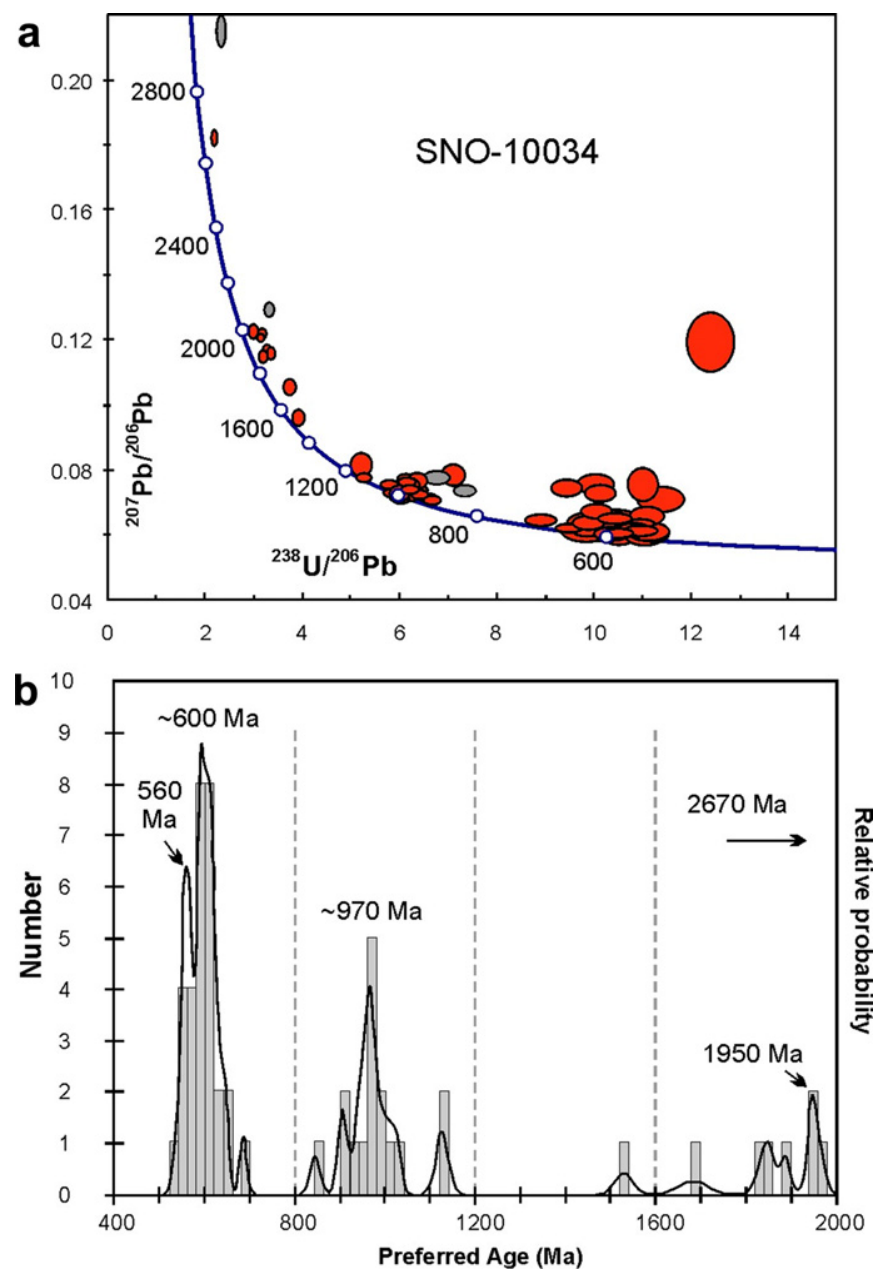

Fig. 8. UePb data for detrital zircon grains from a sample representing the metamorphic basement that hosts the batholith. (a) TeraeWasserburg plot. Note that the data are uncorrected for common $\mathrm{Pb}$ : points that are $>10 \%$ discordant after correction are indicated by grey ellipses (as explained in the text, this was only carried out for data with ages over ca. $800 \mathrm{Ma}$ ). (b) probability diagram showing the frequency of $\mathrm{UePb}$ ages in the detrital zircon population, ignoring the discordant points shown in (a). 


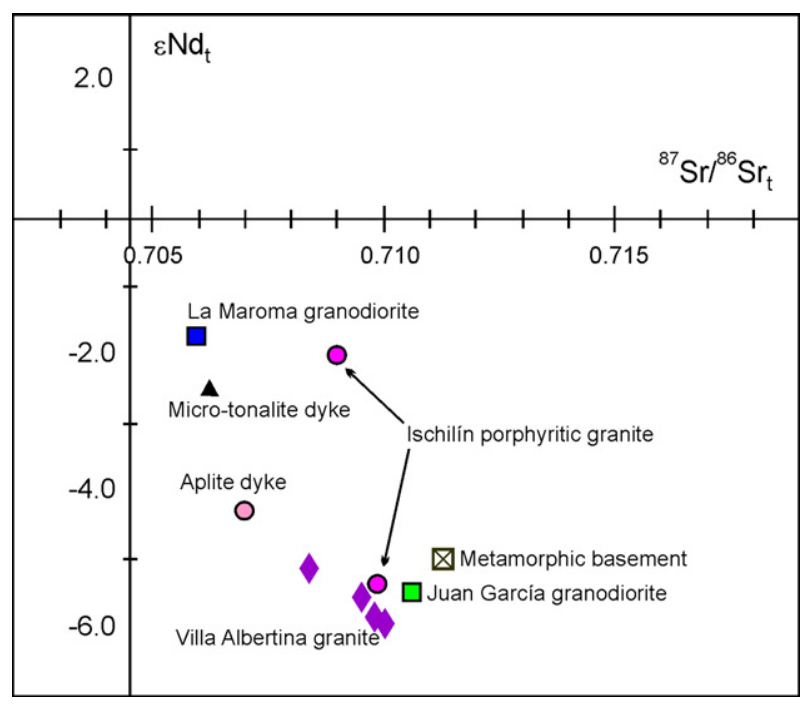
Fig. 9. Diagram of initial $\varepsilon N d$ and ${ }^{87} \mathrm{Sr} /{ }^{86} \mathrm{Sr}$ values, showing a strong crustal signature for
the samples of the southern Sierra Norte-Ambargasta batholith and associated rocks.

The resulting D1 structures are mostly folds around NeS to NE eSW trending axes, and dextral shear on $\mathrm{S} 1$ planes supported by dextral mylonitic shearing in some granites. A second-stage deformation (D2) is also related to dextral shear and the two deformational events seem to belong to a continuous process. It should be noted that Miró et al. (1999) interpreted the foliated granites as having been emplaced syn-kinematically in shear zones.

We consider that the formation of the mylonite belt occurred during D2, the last deformation affecting the area, as no ductile deformation of any sort was recognized in the cross-cutting Villa Albertina granite. In contrast, the main metaluminous granitic suite is considered to be pre-/syn-mylonitic as it shows, in many places, a N E eSW orientation of phenocrysts and mafic enclaves, suggesting that these bodies were still partially molten during an earlier stage of deformational history (D1), and that they were then strongly affected by the D2 mylonitization. This would suggest progression from D1 to D2 in a short period of time, an idea strongly supported by the new $\mathrm{UePb}$ zircon ages.

Our data show that the pre-/syn-mylonitic Juan García granodiorite crystallized at $537 \pm 4 \mathrm{Ma}$. On the other hand, the

Table 6

Summary of UePb zircon geochronology for the Sierra Norte de Córdoba.

\begin{tabular}{|c|c|c|c|}
\hline Unit & Age (Ma) & Method & Reference \\
\hline 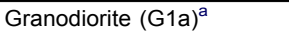 & $533 \pm 2$ & TIMS $^{\mathrm{b}}$ & Rapela et al. (1998a, \\
\hline $\begin{array}{l}\text { Rhyodacite } \\
\text { (Sierra de Ambargasta) }\end{array}$ & $557 \pm 4$ & b) TIMS $^{\text {b }}$ & Söllner et al. (2000) \\
\hline $\begin{array}{l}\text { Ojo de Agua Formation } \\
\text { (Monzogranite) }\end{array}$ & $515 \pm 4$ & SHRIMP & $\begin{array}{l}\text { Stuart-Smith } \\
\text { et al. (1999) }\end{array}$ \\
\hline $\begin{array}{l}\text { Los Burros dacite } \\
\text { (Ambargasta) }\end{array}$ & $\begin{array}{c}512.6 \pm 3.5 \\
481 \pm 1.5 \\
531.6 \pm 1.8\end{array}$ & SHRIMP & Leal et al. (2003) \\
\hline $\begin{array}{l}\text { Ignimbrite in La } \\
\text { Lidia Formation }\end{array}$ & \multicolumn{3}{|c|}{584 (b22/-14) TIMS ${ }^{\text {b }}$ Llambías et al. (2003) } \\
\hline \multicolumn{2}{|c|}{$\begin{array}{l}\text { Undeformed monzogranite } 554 \pm 7 \\
542 \pm 11\end{array}$} & TIMS $^{\mathrm{b}}$ & Schwartz et al. (2008) \\
\hline Undeformed granodiorite 53 & \pm 8 & TIMS $^{\mathrm{b}}$ & Schwartz et al. (2008) \\
\hline Porphyritic dacite & $535 \pm 8$ & TIMS $^{\mathrm{b}}$ & Schwartz et al. (2008) \\
\hline Miariolitic monzogranite & $541 \pm 12$ & TIMS $^{\mathrm{b}}$ & Schwartz et al. (2008) \\
\hline San Miguel gneiss & $\begin{array}{l}533 \pm 12 \\
492.2 \pm 3.6\end{array}$ & SHRIMP & $\begin{array}{l}\text { Siegesmund } \\
\text { et al. (2010) }\end{array}$ \\
\hline Juan García granodiorite & $537 \pm 4$ & SHRIMP & This paper \\
\hline Villa Albertina granite & $530 \pm 4$ & SHRIMP & This paper \\
\hline
\end{tabular}

a Combined result with granite gneiss from Sierra de Cordoba.

b Forced upper intercept ages. undeformed Villa Albertina granite, which cuts the mylonite belt (of which it contains abundant xenoliths), gave an age of $530 \pm$ $4 \mathrm{Ma}$. These ages, which are almost indistinguishable within analytical uncertainty, constrain the entire sequence of metaluminous to peraluminous magmatism and the development of the mylonitic shear belt to a relatively brief period of no more than a maximum of about 10e15 Ma within the Early Cambrian (Terre-neuvian). This marks the end of the Pampean orogeny in this sector of the Eastern Sierras Pampeanas.

The detrital zircon pattern of a quartz-rich metasedimentary host rock to the batholith has major peaks at ca. $560 \mathrm{Ma}$, ca. $600 \mathrm{Ma}$ and ca. $970 \mathrm{Ma}$ (Fig. 8), with the younger detrital peak at $560 \mathrm{Ma}$ indicating that deposition age of the metasedimentary sequence cannot be older than Late Ediacaran. This pattern is very similar to those found in other sectors of the Eastern Sierras Pampeanas (e.g., Schwartz and Gromet, 2004; Rapela et al. 2007; Escayola et al., 2007) and also in the Puncoviscana Formation (e.g. Adams et al., 2011), which is considered the northern low-grade equivalent of the metasediments of Sierras de Córdoba and Sierras Norte de Córdoba (Rapela et al., 1998b; Von Gosen and Prozzi, 2010). A remarkable feature of all these detrital patterns for Pampean belt metasedimentary rocks is the absence of ages in the typical range of the Río de la Plata craton (2050e2250 Ma, Rapela et al., 2007), which is at present the nearest crystalline basement east of Sierra Norte (Fig. 1). This shows that the boundary between the Eastern Sierras Pampeanas and the craton is probably one of transpres-sional tectonic docking during the Early Cambrian: the Punco-viscana Formation and its equivalents cannot represent a passive margin sequence deposited over the edge of the craton.

9.3. Geochemical and isotopic characteristics of the granites and their sources

Previous studies of the granites of the Sierra Norte-Ambargasta batholith indicate that it is dominated by a calc-alkaline, metaluminous to slightly peraluminous suites, inferred to have been associated with a magmatic arc (Bonalumi, 1988; Lira et al., 1997; Gordillo et al., 1997; Rapela et al., 1998b). In the central and northern part of the batholith, the last magmatic phase resulted in volcanic suites (rhyolites, dacites, andesites and basaltic andesites), which also have calc-alkaline signatures (e.g., Leal et al., 2003; Koukharsky et al., 2003). Our geochemical analyses of the intermediate to siliceous $\left(61 \mathrm{e} 77 \% \mathrm{SiO}_{2}\right)$ granitic units from the southern part of the batholith also indicate a dominant calc-alkaline suite, composed of metaluminous to weak peraluminous granites, that may also be ascribed to a convergent margin (Figs. 4e6).

The high initial ${ }^{87} \mathrm{Sr} /{ }^{86} \mathrm{Sr}$ ratios and negative $\varepsilon N d t$ values of both pre-/syn-and post-mylonitic rocks (Fig. 9) are evidence of a high degree of crustal contamination, possibly involving melting and/or hybridization with the metamorphic basement host rocks, with only the La Maroma granodiorite indicating the presence of less contaminated arc magmas. It may be significant that the majority of these bodies were emplaced essentially contemporaneously with strong compressive deformation of Early Cambrian age (D1 and D2 events of Von Gosen and Prozzi (2010)). Crustal contamination is a common feature of granites emplaced in the Neoproterozoic transpressional belts of southwestern Gondwana, e.g., the Dom Feliciano belt of Uruguay and southeast Brazil (Chemale et al., 2012, and references therein). It seems that granite emplacement in these major shear zones active during the assembly of Gondwana favoured an unusual degree of crustal interaction.

Whereas the isotope compositions point to the local metamorphic basement as a likely immediate source of the crustal signature of the batholithic magmas, the SmeNd model ages give some idea of the ultimate provenance of such metasedimentary 
material. The rather uniform $\mathrm{T}_{\mathrm{DM}}$ ages of $1400 \mathrm{e} 1600 \mathrm{Ma}$ (Table 5) is an indication of the recycling of mature crustal material in the sedimentary environment, which is a common characteristic of Pampean magmatic rocks. Pankhurst et al. (1998), Rapela et al. (1998b) and Escayola et al. (2007) inferred the age of the source to be slightly older (Paleoproterozoic, between 1600 and $1700 \mathrm{Ma}$ ). However, it is a general conclusion, re-emphasized here, that they could not have derived from material separated from the mantle before $1800 \mathrm{e} 1900 \mathrm{Ma}$, such as the Paleoproterozoic rocks of the Río de la Plata craton $(2000 \mathrm{e} 2250 \mathrm{Ma})$. According to Rapela et al. (1998b) and Escayola et al. (2007), the magmas that formed the Cambrian plutons of Sierra Chica and Sierra Grande de Córdoba had sources derived from the mantle in two periods: at the end of the Paleoproterozoic (1800e1600 Ma) and during the Mesoproterozoic (1600e1100 Ma), the former being the more important.

\subsection{Timing of events along the Pampean belt and geodynamic scenario}

Geochronological results presented in this study impose important constraints to the timing of sediment deposition and the main deformation in Sierra Norte de Córdoba. The youngest peak of detrital zircon ages in the metasedimentary host rocks demon-strate that original sedimentation could not have been older than c. $560 \mathrm{Ma}$. At the same time they may have been intruded by earlier calc-alkaline granites at $554 \pm 7$ ( Schwartz et al., 2008, see Table 6 and Section 9.1). It is thus probable that deposition of the sediments was synchronous with the early stages of arc magmatism. A recent study at the type section of the Puncoviscana Fm, in the northern part of the Pampean Belt, shows that magmatic arc activity was in fact coeval with deposition of turbiditic deposits at $537 \pm 1 \mathrm{M} \mathrm{a}$ (Escayola et al., 2011).

Although there is no precise age constraint for the beginning of the compressive D1 event in the Sierra Norte de Córdoba, dextral transpression was active at the time of emplacement of the $537 \pm 4$ Ma mylonitized Juan García granodiorite, and was complete before $530 \pm 4 \mathrm{Ma}$ when the Villa Albertina granite was emplaced. Therefore, the onset of crustal thickening in this part of the Pampean orogen most probably occurred rather earlier than previous estimates (e.g., 520e530 Ma, Rapela et al., 1998b).

To the S and SSW of the study area, the Sierras de Córdoba (Fig. 1) are dominated by highly peraluminous rocks: extensive migmatitic massifs of cordierite and garnetecordierite migmatites, cordierite granites and also a cordieritite complex (e.g., Gordillo, 1984; Rapela et al., 1998a, 2002; Otamendi and Patiño Douce, 2001). Peak metamorphism in these rocks has been estimated by $\mathrm{UePb}$ dating of monazites in garnetecordierite migmatites at $522 \pm 8$ $\mathrm{Ma}$ and zircons in cordierite granites at $523 \pm 2 \mathrm{M}$ a (Rapela et al., 1998b). Thus widespread peraluminous magmatism and crustal anatexis in the Pampean orogen reached its peak as much as $14 \pm 5$ Ma after the dextral compressive events had come to an end in the Sierra Norte de Córdoba, i.e., between $537 \pm 4 \mathrm{Ma}$ and $530 \pm 4 \mathrm{Ma}$.

This strong peraluminous terrain of the Sierras de Córdoba has been postulated as a result of the accretion of a continental fragment, the Pampean terrane, in a collisional setting during the Early Cambrian, that was preceded by eastward-dipping subduction (Rapela et al., 1998b). The Sierra Norte area would have been part of the magmatic arc in the upper plate, while the strongly peraluminous belt of the Sierras de Córdoba in the west (Fig. 1) i s interpreted as the accretionary complex formed on the lower plate (Schwartz et al., 2008; Von Gosen and Prozzi, 2010). It is though that the collision was dextral and oblique, and the colliding ("Pampean") terrane was part of the Mesoproterozoic ArequipaeAntofalla and Western Sierras Pampeanas blocks (Rapela et al., 2007), located to the west. Other authors also favour a Late EdiacaraneEarly Cambrian magmatic arc, but propose a noncollisional setting for the strong peraluminous terrains, such as active ridge subduction (Schwartz et al., 2008 and references therein). Major problems related with the terrane collision model raised by these authors are (a) the time lapse between the convergence and thickening of continental crust and the generation of peraluminous magmatism is too short to explain anatexis by conductive heating, and (b) the spatial separation between the calc-alkaline arc and the peraluminous terrain.

As discussed above, the new evidence presented allows a lapse of up to $14 \pm 5 \mathrm{Ma}$ between crustal thickening and the peak of peraluminous magmatism in the study area and the northeastern part of the Sierras de Córdoba, a period that is comparable to the minimum lag time of $10 \mathrm{e} 20 \mathrm{Ma}$ observed in modern continental collisions such as the Alps (Von Blanckenburg and Davies, 1995) and the Himalaya (Kohn and Parkinson, 2002) orogenies. Moreover, as pointed out by Escayola et al. (2011), the ridge subduction model cannot explain the presence of Early Cambrian (523 Ma) plutons similar to those of the Eastern Sierras Pampeanas in the Antofalla block. In the northern sector of the Pampean belt, the folded turbidites were deposited at $537.3 \pm 0.9 \mathrm{Ma}$ (Early Cambrian, the age of interbedded felsic tuffs), with deformation inferred to have occurred in the interval 537e524 Ma (Escayola et al., 2011). Thus deposition of the sediments in the south appears to have started earlier (>555 Ma, Late Neoproterozoic). Although deformation occurred in the Early Cambrian all along the Pampean belt, compressive deformation had already started at the time of intru-sion of synkinematic granites at $538 \pm 3 \mathrm{Ma}$ in the study area, while the northern sector was still at the sedimentation stage. These observations would be compatible with the idea that the Pampean orogeny was diachronous, progressing from south to north during the Late Ediacaran to Early Cambrian times. It should be noticed, that two rather imprecise UePb SHRIMP zircon ages for high-grade metamorphic rocks in the southeastern sectors of Sierra de Córdoba and Sierra de San Luis, suggest an even earlier Neoproterozoic history, still poorly understood $(577 \pm 11 \mathrm{Ma} ; 564 \pm 21 \mathrm{Ma}$, Siegesmund et al., 2010).

We suggest that the new evidence obtained in the southern sector of the Sierra Norte-Ambargasta batholith are better explained by a geodynamic model involving intrusion of a Late Neoproterozoic to Early Cambrian (554e530 Ma) calc-alkaline suite of granites. The initial magmatism was probably related to NE-directed oblique subduction and at $537 \pm 4$ Ma was syntectoni-cally emplaced during folding and crustal thickening with a dextral component (D1). The locus of intrusion evolved to a dextral transpressional belt during this event and the process continued with development of large, dextral mylonitic belts (D2), intruded in turn by $530 \pm 4$ Ma peraluminous granites. The Pampean orogeny culminated with development of widespread strongly per-aluminous migmatitic massifs and emplacement of cordierite granites in the Sierra de Córdoba, to the south of the study area, with a ca. 522 Ma peak metamorphism (P 1/4 $8.6 \pm 0.8 \mathrm{kbar}, \mathrm{T} 1 / 4810 \pm 50{ }^{\circ} \mathrm{C}$, Rapela et al., 1998b) terminated by $515 \mathrm{Ma}$ (Schwartz et al., 2008). The whole Pampean episode involved a Late EdiacaraneEarly Cambrian continental arc, followed in the Early Cambrian by a dextral oblique collision with a string of Meso-proterozoic exotic terranes located to the west (Antofalla and Western Sierras Pampeanas blocks, Rapela et al., 2007; Siegesmund et al., 2010; Escayola et al., 2011; Casquet et al., 2012). Terrane collision followed by slab break-off, may explain also the wide-spread crustal anatexis and emplacement of $520 \pm 3 \mathrm{Ma}$ OIB-like mafic magmas observed in the Sierras de Córdoba (Tibaldi et al., 2008), but the available geological evidence is very scarce to discriminate among different terrane collision models. 
Finally, the absence from the metamorphic envelope of the batholith of detrital zircons of the Río de la Plata craton age indicate that this Paleoproterozoic craton was not the source of sediments of the Pampean belt, which were deposited only shortly before arc magmatism. Large-scale dextral displacement of the Pampean belt during the Early Cambrian was proposed by Rapela et al. (2007) to explain this absence, while Verdecchia et al. (2011) inferred that the craton reached its current relative position in the Mid- to Late Cambrian. The results of this study imply that the dextral displacement took place during the D2 mylonitic event, in the ca. 537 e530 Ma interval.

\section{Conclusions}

New evidence presented in this study of the southern sector of the Sierra Norte-Ambargasta batholith, together with recent structural and geochronological evidence from the Sierra Norte de Córdoba, indicates that this large batholith was emplaced in a transpressional tectonic environment. Plutonic units are distin-guished according to their intrusive relationships with the main deformation events recognized in Sierra Norte, in particular with the penetrative second deformation (D2) that developed the Sauce Puncoelschilín mylonitic belt. Pre-/synmylonitic plutons of met-aluminous to weakly peraluminous granites dominate the lithology of the batholith. Synkinematic granites of this group, were emplaced during a dextral compressive deformation (D1) at $537 \pm 4 \mathrm{Ma}$. The age of Sauce Puncoelschilín mylonitic belt, that affected all previous units, is constrained between this age and one of $530 \pm 4 \mathrm{Ma}$ age for a post-mylonitic leucocratic granite.

A model involving oblique subduction followed by oblique dextral collision with a continental terrane, could explain the observed Late EdiacaraneEarly Cambrian calc-alkaline magmatism, dextral transpression and crustal thickening $(>530$ $\pm 4 \mathrm{Ma}$ ) and development of widespread anatexis, peraluminous magmatism and peak metamorphism to the SW in the Sierras de Córdoba at 522e515 Ma (Rapela et al., 1998b). Deformation in the Pampean belt may have been diachronous, progressively younger towards the north, which is consistent with the postulated model of dextral oblique collision with a continental terrane. A large dextral displacement of the Pampean belt relative to the Río de la Plata craton took place during the D2 mylonitic event.

\section{Acknowledgements}

Financial support for this paper was provided by PIP-CONICET 01940; FONCYT, BID 1728 OC/AR, PICT-1009 (Argentina) and CGL2009-07984/BTE (Spain). The first author gives special thanks to Udo Zimmerman for his cordiality during a study visit to Johannesburg University, South Africa and to the inhabitants of the mountains that allowed us access to the outcrops. We appreciate constructive reviews by $M$. López de Luchi of an earlier version of the manuscript and thorough reviews and in-depth comments made by Juan Otamendi and an anonymous reviewer.

\section{References}

Aceñolaza, F.G., Miller, H., Toselli, A.J. (Eds.), 1990. El Ciclo Pampeano en el Noroeste Argentino. Serie Correlación Geológica, vol. 4. Universidad Nacional de Tucumán.

Adams, C.J., Miller, H., Aceñolaza, F.G., Toselli, A.J., Griffin, W.L., 2011. The Pacific Gondwana margin in the late Neoproterozoic-early Paleozoic: detrital zircon U-Pb ages from metasediments in northwest Argentina reveal their maximum age, provenance and tectonic setting. Gondwana Research 19, 71e83.

Baldo, E.G., Pankhurst, R.J., Rapela, C.W., Saavedra, J., Mazieri, C., 1998. Granito “El Cerro", Magmatismo Colisional Famatiniano en el Sector Austral de la Sierra Norte-Ambargasta, Córdoba. In: Actas X Congreso Latinoamericano de Geología y VI Congreso Nacional de Geología Económica, vol. II, pp. 374e378.
Bonalumi, A.A., 1988. Características petrológicas y geoquímicas de los granitoides asociados a la mineralización de manganeso en el norte de la provincia de Córdoba y sur de Santiago del Estero, República Argentina. In: 5th Congreso Geológico Chileno, Santiago, Actas, vol. 2, pp. E 47eE 61.

Caminos, R., 1972. Sierras Pampeanas de Catamarca, Tucumán, La Rioja y San Juan. In: Leanza, A.F. (Ed.), II Simposio de Geología Regional Argentina, vol. 1. Academia Nacional de Ciencias de Córdoba, pp. 41 e79.

Candiani, J.C., Stuart-Smith, P., Gaido, F., Carignano, C., Miró, R., López, H., 2001.

Hojageológica 3163-I: Jesús María, Provincia de Córdoba. SEGEMAR, Buenos Aires, Boletín 314

Casquet, C., Rapela, C.W., Pankhurst, R.J., Baldo, E.G., Galindo, C., Fanning, C.M., Dahlquist, J.A., Saavedra, J., 2012. A history of Proterozoic terranes in southern South America: from Rodinia to Gondwana. Geoscience Frontiers 2, 137e145.

Castellote, P., 1982. Estudio geológico y geocronológico del basamento cristalino

del sector septentrional de la Sierra Norte de Córdoba. Acta Geológica Lilloana $17,61 \mathrm{e} 66$.

Chemale Jr., F., Mallmann, G., Bitencourt, M.F., Kawashita, K., 2012. Time constraints on magmatism along the major Gercino shear zone, southern Brazil: implications for west Gondwana reconstruction. Gondwana Research 22, 184e199.

Dahlquist, J.A., Pankhurst, R.J., Rapela, C.W., Casquet, C., Fanning, C.M., Alasino, Baez, M., 2006. The San Blas PlutorR. Un example of Carboniferous plutonism in the Sierras Pampeanas, Argentina. Journal of South American Earth Sciences 20, 341e350.

DePaolo, D.J., Linn, A.M., Schubert, G., 1991. The continental crustal age distribution: methods of determining mantle separation ages from Sm-Nd isotopic data and application to the southwestern United States. Journal Geophysical Research B96, 2071e2088.

Didier, J., Barbarin, B., 1991. Enclaves and granite petrology. Developments in Petrology 13, 253e261. Elsevier, Amsterdam.

Escayola, M.P., Pimentel, M.M., Armstrong, R., 2007. Neoproterozoic backarc

basißènsitive high resolution ion microprobe $\mathrm{U}-\mathrm{Pb}$ and $\mathrm{Sm}-\mathrm{Nd}$ isotopic evidence from the Eastern Pampean Ranges, Argentina. Geology 35, 495e498. Escayola,

M.P., van Staal, C.R., Davis, W.J., 2011. The age and tectonic setting of the Puncoviscana Formation in northwestern Argentina: an accretionary complex related to Early Cambrian closure of the Puncoviscana Ocean and accretion of the Arequipa-Antofalla block. Journal of South American Earth Sciences 32, 438e459.

Frost, B.R., Barnes, C.G., Collins, W.J., Arculus, R.J., Ellis, S.J., Frost, C.D., 2001. A geochemical classification for granitic rocks. Journal of Petrology 42, $2033 e 2048$.

González, R.R., Cabrera, M.A., Bortolotti, P., Castellote, P., Cuenya, M.P., Omil,

D.M., Moyano, R.O., Ojeda, J.R., 1985. La actividad eruptiva en Sierras Pampeanas. Esquematización geográfica y temporal. Acta Geológica Lilloana XVI, 289e318.

Gordillo, C.E., 1984. Migmatitas cordieríticas de la Sierras de Córdoba; condiciones físicas de la migmatización. Boletín de la Academia Nacional de Ciencias, Córdoba 68 , 3e40.

Gordillo, D.E., 1996. Granitoides del sector sudeste de la Sierra de Ischilín.

Depermento de Ischilín-Córdoba. Tesis de Licenciatura, Universidad Nacional de Córdoba, Argentina.

Gordillo, D.E., Kirschbaum, A.M., Baldo, E., 1997. Magmatismo del Borde Oriental de las Sierras Pampeanas: los granitoides de la Sierra de Ischilín, Provincia de Córdoba, Argentina. In: 8th Congreso Geológico Chileno, Antofagasta, Actas, vol. II, pp. $1290 \mathrm{e} 1294$.

Gordillo, C.A., Lencinas, A.N., 1979. Sierras Pampeanas de Córdoba y San Luis. In: Turner, J.C. (Ed.), Segundo Simposio de Geología Regional Argentina, vol. 1. Academia Nacional de Ciencias, Córdoba, pp. 577e650.

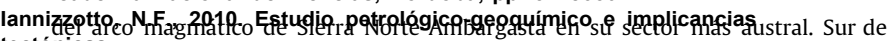

tectónicaśerra Norte de Córdoba. Unpublished PhD thesis. (1087), Universidad Nacional de La Plata, Facultad de Ciencias Naturales y Museo.

lannizzotto, N.F., Rapela, C.W., Baldo, E.G.A., 2011. Nuevos datos geocronológicos, geoquímicos e isotópicos del Batolito de Sierra Norte-Ambargasta en su sector más austral, Provincia de Córdoba. In: XVIII Congreso Geológico Argentino, Mayo, Neuquén, Actas del Simposio de Tectónica Pre-Andina (S2), pp. 190e191.

Kirschbaum, A.M., Pérez, M.B., Baldo, E.G., Gordillo, D.E., 1997. Magmatismo Oriental de las Sierras Pampeanas de Córdoba, Argentina: Petrografia y Geoquímica de los Granitoides de la Sierra de Macha. In: VIII Congreso Geológico Chileno, Antofagasta, Actas, vol. II, pp. 1319e1323.

Kohn, M.J., Parkinson, C.D., 2002. Petrologic case for Eocene slab break off during the Indo-Asian collision. Geology 30, 591e594.

Koukharsky, M., Brodtkorb, M.K., de Kay, S.M., Munizaga, F., 2003. La Formación

Balbuena, integrante del arco magmático pampeano en la sierra de Ambargasta, provincia de Santiago del Estero. Revista de la Asociación Geológica Argentina 58, $583 e 592$

Kretz, R., 1983. Symbols for rock-forming minerals. American Mineralogist 68, $277 \mathrm{e} 279$.

Leal, P.R., Hartmann, L.A., Santos, J.O.S., Miró, R.C., Ramos, V.A., 2003. Volcanismo

postorogénico en el extremo norte de la Sierras Pampeanas Orientales: nuevos datos geocronológicos y sus implicancias tectónicas. Revista de la Asociación Geológica Argentina 58, 593e607.

granitoid activity in the Sierra Norte-Ambargasta Ranges, Central Argentina.

Lira, R., Millone, H.A., Kirschbaum, A.M., Moreno, R.S., 1997. Calc-alkaline arc Journal of South American Earth Sciences 10, 157e177.

Llambías, E.J., Gregori, D., Basei, M.A., Varela, R., Prozzi, C., 2003. Ignimbritas riolíticas neoproterozoicas en la Sierra Norte de Córdoba: ¿evidencia de un arco 
magmático temprano en ciclo Pampeano? Revista de la Asociación Geológica Argentina $58,572 \mathrm{e} 582$.

Lucero Michaut, H.N., 1969. Descripción geológica de las hojas 16h, Pozo Grande y 17h, Chuña Huasi, Provincias de Córdoba y Santiago del Estero, Carta GeológicoEconómica de la República Argentina, scale 1:200.000, Ministerio de Economía y Trabajo, Dirección Nacional de Geología y Minería, Buenos Aires, Boletín 107, pp. $1 \mathrm{e} 28$.

Lucero Michaut, H.N., 1979. Sierras Pampeanas del Norte de Córdoba, Sur de Santiago del Estero, Borde Oriental de Catamarca y Angulo Sudeste de Tucumán. In: Segundo Simposio de Geología Regional Argentina, Academia Nacional de Ciencias, Córdoba, vol. 1, pp. 293e347.

Ludwig, K.R., 2001. SQUID 1.02, a User's Manual. Berkeley Geochronology Center Special Publication No. 2, 2455 Ridge Road, Berkeley, CA 94709, US.

Ludwig, K.R., 2003. Isoplot/Ex Version 3.0: a Geochronological Toolkit for Microsoft Excel. Berkeley Geochronology Center Special Publication No. 4, 2455 Ridge Road, Berkeley CA 94709, USA.

Lyons, P., Skirrow, R.G., Stuart-Smith, P.G., 1997. Informe geológico y metalogénico de las Sierras Septentrionales de Córdoba (Provincia de Córdoba). scale 1: 250000. Anales XXVII, Instituto de Geología y Recursos Minerales SEGEMAR, Buenos Aires.

Martino, R.D., 2003. Las fajas de deformación dúctil de las Sierras Pampeanas de Córdoba: Una reseña general. Revista de la Asociación Geológica Argentina 58, $549 \mathrm{e} 571$.

Martino, R.D., Painceyra, R.A., Guereschi, A.B., Sfragulla, J.A., 1999a. Transcurrencia paralela al arco magmático de la sierra Norte de Córdoba: la faja de deformación de Sauce Punco. In: 14th Congreso Geológico Argentino. Actas, vol. 1, p. 37.

Martino, R.D., Painceyra, R., Guereschi, A., Sfragulla, J., 1999b. La faja de deformación de Sauce Punco, Sierra Norte, Córdoba, Argentina. Revista de la Asociación Geológica Argentina 53, 436e440.

Massabie, A., Mutti, D., Nestiero, O., 2002. Edad, afinidades geoquímicas y tectónicas del pórfido riolítico de La Lidia, Sierra Norte de Córdoba. Revista de la Asocia-ción Geológica Argentina 57, 80e84.

Methol, E.J., 1958. Descripción Geológica de la Hoja 18i (Deán Funes) Córdoba. Boletín Dirección Nacional de Geología y Minería 88. Buenos Aires.

Miró, R.C., Gaido, M.F., Candiani, J.C., 1999. Fajas de deformación del batolito Sierra Norte de Córdoba y Santiago del Estero. In: XIV Congreso Geológico Argentino Actas T1, pp. 152e155.

Miró, R.C., Sapp, M., 2001. Hoja Geológica 2963-III. Villa Ojo de Agua, Provincia de Santiago del Estero y Córdoba. SEGEMAR Boletín 315.

Nakamura, E., 1974. Determination of REE, Ba, Fe, Mg, $\mathrm{Na}$ and $\mathrm{K}$ in carbonaceous and ordinary chondrites. Geochimica et Cosmochimica Acta 38, 757e773. Omarini, R.H.

Sureda, R., Toselli, A.J., Rossi, J.N., 1999. Magmatismo. In: González Bonorino, G., Omrini, R., Viramonate, J. (Eds.), Relatorio del XIV Congreso Geológico Argentino, Tomo I, pp. 29e40.

Otamendi, J.E., Patiño Douce, A.E., 2001. Partial melting of aluminous metagreywackes in the northern Sierra de Comechingones, Central Argentina. Journal of Petrology 42, 1151e1172.

Pankhurst, R.J., Rapela, C.W., 1998. The proto-Andean margin of Gondwana: an introduction. In: Pankhurst, R.J., Rapela, C.W. (Eds.), The Proto-Andean Margin of Gondwana, vol. 142. Geological Society, London, Special Publication, pp. 1e9.

Pankhurst, R.J., Rapela, C.W., Saavedra, J., Baldo, E., Dalquist, J., Pascua,

L., Fanning, C.M., 1998. The Famatinian magmatic arc in the central Sierras Pampeanas: an Early to Mid-Ordovician continental arc on the Gondwana margin. In: Pankhurst, R.J., Rapela, C.W. (Eds.), The Proto Andean Margin of Gondwana, vol. 142. Geological Society, London, Special Publication, pp. 343e367.

Pastore, F., Methol, E., 1953. Descripción geológica de la hoja 19i, Capilla del Monte. Provincia de Córdoba. Boletín Dirección Nacional de Geología y Minería, SEGEMAR, Buenos Aires 79, 1e70

Rapela, C.W., Bonalumi, A., Baldo, E., 1991. Edad y geoquímica del Pórfido Riolítico de Oncán, Sierra Norte de Córdoba, Sierras Pampeanas, Argentina, Congreso Geológico Chileno, 1991. Resúmenes ampliados, pp. 19e22.

Rapela, C.W., Pankhurst, R.J., Casquet, C., Baldo, E., Saavedra, J., Galindo, C., Fanning, C.M., 1998a. The Pampean Orogeny of the Southern proto-Andes: Cambrian continental collision in the Sierras de Córdoba. In: Pankhurst, R.J., Rapela, C.W. (Eds.), The Proto-Andean Margin of Gondwana, vol. 142. Geological Society, London, Special Publication, pp. 181e217.

Rapela, C.W., Pankhurst, R.J., Casquet, C., Baldo, E., Saavedra, J., Galindo, C.,1998b. Early evolution of the proto-Andean margin of South America. Geology 26, 707e710.

Rapela, C.W., Baldo, E.G., Pankhurst, R.J., Saavedra, J., 2002. Cordieritite and leucogranite formation during emplacement of highly peraluminous magma: the El Pilón granite complex (Sierras Pampeanas, Argentina). Journal of Petrology 43, 1003 e1028.
Rapela, C.W., Pankhurst, R.J., Casquet, C., Fanning, C.M., Baldo, E., González Casado, J.M., Galindo, C., Dahlquist, J., 2007. The Río de La Plata craton and the assembly of SW Gondwana. Earth-Science Reviews 83, 49e82.

Rapela, C.W., Baldo E., Pankhurst, R.J., Fanning C.M., 2008. The Devonian Achala batholith of the Sierras Pampeanas: F-Rich, aluminous A-Types granites. In: VI South American Symposium on Isotope Geology, San Carlos de Bariloche, Argentina, CD-ROOM, $8 \mathrm{pp}$

Rapela, C.W., Pankhurst, R.J., Casquet, C., Baldo, E., Galindo, C., Fanning, C.M., Dahlquist, J.M., 2010. The Western Sierras Pampeanas: protracted Grenville-age history (1330-1030 Ma) of intra-oceanic arcs, subduction-accretion at continental-edge and AMCG intraplate magmastism. Journal of South American Earth Sciences 29, 105e127.

Sato, A.M., González, P.D., Llambías, E.J., 2003. Evolución del Orógeno Famatiniano en la Sierra de San Luis: magmatismo de arco, deformación y metamorfismo de bajo a alto grado. Revista de la Asociación Geológica Argentina 58, 487e504.

Schwartz, J.J., Gromet, P.L., 2004. Provenance of a late Proterozoic-early Cambrian basin, Sierras de Córdoba, Argentina. Precambrian Research 129, 1e21.

Schwartz, J., Gromet, L., Miro, R., 2008. Timing and duration of the calc-alkaline arc of the Pampean orogeny implications for the Late Neoproterozoic to Cambrian evolution of Western Gondwana. Journal of Geology 116, 39e61. Siegesmund, S.

Steenken, A., Martino, R.D., Wemmer, K., López de Luchi, M.G., Frei, R., Presnyakov, S., Guereschi, A., 2010. Time constraints on the tectonic evolution of the Eastern Sierras Pampeanas (Central Argentina). International Journal of Earth Sciences 99, 1199e1226.

Söllner, F., Leal, P.R., Miller, H., Brodtkorb, M., 2000. Edades U/Pb en circones de la riodacita de la sierra de Ambargasta. Provincia de Córdoba. Mineralogía y Metalogenia. In: Schalamuk, I., Brodtkorb, M., Etcheverry, R. (Eds.), La Plata, pp. 465e469. Publicación 6 .

Steenken, A., López de Luchi, M.G., Siegesmund, S., Wemmer, K., Pawlig, S., 2004. Crustal provinces and cooling of the basement complexes of the Sierra de San Luis: an insight into the tectonic history of the proto-Andean margin of Gondwana. Gondwana Research 7, 1171e1195.

Streckeisen, A., 1976. To each plutonic rock its proper name. Earth-Science Reviews 13, 1 e33.

Stuart-Smith, P.G., Camacho, A., Sims, J.P., Skirrow, R.G., Lyons, P., Pieters,

P.E.Black, L.P., 1999. Uranium-lead dating of felsic magmatic cycles in the southern Sierras Pampeanas, Argentina: implications for the tectonic development of the proto-Andean Gondwana margin. In: Ramos, V.A., Keppie, J.D. (Eds.) Laurentia-Gondwana Connections before Pangea. GSA, Special Paper, 336, pp. $87 \mathrm{e} 114$

Thompson, R.N., 1982. British tertiary volcanic province. Scottish Journal of Geology $18,49 \mathrm{e} 107$.

Tibaldi, A.M., Otamendi, J.E., Gromet, L.P., Demichelis, A.H., 2008. Suya Taco and Sol de Mayo mafic complexes from Eastern Sierras Pampeanas, Argentina: evidence for the emplacement of primitive OIB-like magmas into deep crustal levels at the late stage of the Pampean orogeny. Journal of South American Earth Sciences 26, $172 \mathrm{e} 187$.

Toselli, A.J., 1990. Metamorfismo del Ciclo Pampeano. In: Aceñolaza, F.G., Miller, H., Toselli, A.J. (Eds.), El Ciclo Pampeano en el Noroeste Argentino. Serie Correlación Geológica, vol. 4. Universidad Nacional de Tucumán, pp. 181e198.

Turner, J.C.M., 1960. Estratigrafía de la Sierra de Santa Victoria y adyacencias. Academia Nacional de Ciencias Córdoba, Boletín 41, 163e196.

Verdecchia, S.O., Casquet, C., Baldo, E.G., Pankhurst, R.J., Rapela, C.W., Fanning, C.M., Galindo, C., 2011. Docking of the Río de la Plata craton to southwestern Gondwana: age constraints from U-Pb SHRIMP detrital zircon ages from Sierras de Ambato and Velasco (Sierras Pampeanas, Argentina). Journal of the Geological Society, London 168, 1061e1071.

Vernon, R.H., 1984. Microgranitoid enclaves in granites-globules of hybrid magma quenched in a plutonic environment. Nature $309,438 \mathrm{e} 439$.

Von Blanckenburg, F., Davies, J.H., 1995. Slab break off: a model for syncollisional magmatism and tectonics in the Alps. Tectonics 14, 120e131.

Von Gosen, W., Prozzi, C., 2010. Pampean deformation in the Sierra Norte de Córdoba, Argentina: implications for the collisional history at the western preAndean Gondwana margin. Tectonics 29, 1 e33.

Williams, I.S., 1998. U-Th-Pb geochronology by ion microprobe. In: McKibben, M.A. Shanks, W.C., Ridley, W.I. (Eds.), Applications of Microanalytical Techniques to Understanding Mineralizing Processes. Reviews of Economic Geology, vol. 7, pp. $1 \mathrm{e} 35$.

Willner, A.P., 1990. División tectonometamórfica del basamento del noroeste Argentino. In: Aceñolaza, F.G., Miller, H., Toselli, A.J. (Eds.), El Ciclo Pampeano en el Noroeste Argentino, Universidad Nacional de Tucumán, Serie Correlación Geológica 4, pp. 113e159 